\title{
Geometric characterizations of Gromov hyperbolicity $^{\star}$
}

\author{
Zoltán M. Balogh ${ }^{1}$, Stephen M. Buckley ${ }^{2}$ \\ 1 Departement Mathematik, Universität Bern, Sidlerstrasse 5, 3012, Bern, Schweiz \\ (e-mail: zoltan@math-stat.unibe.ch) \\ 2 Department of Mathematics, National University of Ireland, Maynooth, Co. Kildare, \\ Ireland (e-mail: sbuckley@maths .may.ie)
}

Oblatum 17-I-2002 \& 9-XII-2002

Published online: 10 April 2003 - (C) Springer-Verlag 2003

\begin{abstract}
We prove the equivalence of three different geometric properties of metric-measure spaces with controlled geometry. The first property is the Gromov hyperbolicity of the quasihyperbolic metric. The second is a slice condition and the third is a combination of the Gehring-Hayman property and a separation condition.
\end{abstract}

\section{Introduction}

The purpose of this paper is to show that three (apparently) different geometric properties of Euclidean domains (and more general metric spaces) are in fact equivalent. These properties have various far-reaching analytical consequences. The very different nature of these applications serves as motivation for proving this equivalence.

The first such property is the so-called Gromov hyperbolicity. This expresses the property of a general metric space to be "negatively curved" in the sense of coarse-geometry. The importance of Gromov hyperbolicity is widely appreciated. This notion was introduced by Gromov in the setting of geometric group theory [Gr1], [Gr2], [GhHa] but has played an increasing role in analysis on general metric spaces [BoHeKo], [BoSc] with applications to the Martin boundary, invariant metrics in several complex variables [BaBo1], [BaBo2] and extendability of Lipschitz mappings [Ln].

* This research was partially supported by the Swiss Nationalfond and Enterprise Ireland. It was partly conducted during two visits by the second author to the University of Bern; the hospitality of the Mathematics Department on both occasions was much appreciated. 
The second property is related to a decomposition of a metric space in subdomains with nice geometry called "slices". The slicing condition was used in Euclidean domains for Sobolev imbedding results (see [BuKo1], [BuKo2], [BuOS], [BuSt1]) and for studying quasiconformal images of various classes of domains [BuSt2].

The third property has its origins in geometric function theory. It is a combination of the so-called Gehring-Hayman condition and a separation condition. The former property was proven in [GeHa] for the case of the hyperbolic metric on simply connected plane domains and more recently in [HeRo] for the case of the quasihyperbolic metric in Euclidean domains that are quasiconformally equivalent to uniform domains. This property is the expression of an interplay between the hyperbolic and Euclidean metric. It says that if the domain is nice enough then the hyperbolic geodesics are quasigeodesics for the Euclidean metric as well. This has numerous applications related to lengths of radii or boundary behavior of the conformal and quasiconformal mappings [BoKoRo], [BaBo3] [HeRo]. The Gehring-Hayman condition for more general metric spaces was recently considered by Bonk-Heinonen-Koskela in [BoHeKo] where it was indicated that Gromov hyperbolicity implies this condition and also a separation condition. The starting point of this paper was a question raised in $[\mathrm{BoHeKo}]$ about the converse of this implication. Our results include the positive answer to this question. The "slice condition" appears as an intermediate step in proving this implication. A side-benefit of the equivalence is the fact that the Gehring-Hayman and separation conditions are potentially much easier to verify for a given domain than the more complicated Gromov hyperbolicity and slice conditions. In view of [BuKo1], [BuKo2], [BuOS], [BuSt1], [BuSt2] our results show an unexpectedly rich analytic content of Gromov hyperbolicity in Euclidean domains.

To state our results precisely let us introduce some notation and terminology. Given a metric space $(X, d)$, a subset $S$ of $X$, and points $x, y \in X$, we write $\operatorname{len}_{d}(S)$ for the length (meaning 1-dimensional Hausdorff measure $\mathcal{H}_{d}^{1}$ ) of $S$, and $\operatorname{dia}_{d}(S)$ for its diameter. Assuming additionally that $x, y \in S$, we use $\Gamma(x, y ; S)$ to denote the class of all rectifiable paths $\gamma:[0, t] \rightarrow S$ for which $\gamma(0)=x$ and $\gamma(t)=y$. We also write $\Gamma(x, y)=\Gamma(x, y ; X)$.

Assuming $X$ is rectifiably connected (i.e. each family $\Gamma(x, y)$ is nonempty), and $\rho: X \rightarrow(0, \infty)$ is a continuous density, then $\left(X, d_{\rho}\right)$ is a metric space where $d_{\rho}$ is defined by the equation

$$
d_{\rho}(x, y)=\inf _{\gamma \in \Gamma(x, y)} \int_{\gamma} \rho(z)|d z|,
$$

where $|d z|$ denotes the length element for the metric $d$. In particular, we denote $d_{\rho}$ by $l$ in the special case $\rho \equiv 1$; we call $l$ the inner metric associated with $d$. Then $(X, d)$ is said to be a length space (in the sense of Gromov) if $l=d$; in this case, $d$ is said to be a length metric or inner metric. 
A metric space $(X, d)$ is geodesic if every pair of points $x, y$ can be joined by a geodesic, that is a path whose length equals the distance from $x$ to $y$. Of course geodesic spaces are length spaces, but the converse is false (consider Euclidean space with the origin removed). If $(X, d)$ is locally compact and the identity map $(X, d) \rightarrow(X, l)$ is continuous (and so a homeomorphism), it is shown in [BoHeKo, Lemma 2.6] that the length of a rectifiable path $\gamma$ in $\left(X, d_{\rho}\right)$ is given by the line integral

$$
\operatorname{len}_{\rho}(\gamma)=\int_{\gamma} \rho(z)|d z|
$$

Thus every such $d_{\rho}$ is a length metric.

Let us pause to mention two important special cases of metrics $d_{\rho}$. First, if $(X, d)$ is Euclidean space, and $\rho(z)=2 /\left(1+|z|^{2}\right)$, then $d_{\rho}$ is the spherical metric on $\mathbb{R}^{n}$, and the Riemann sphere $\overline{\mathbb{R}^{n}}$ is the metric completion of $\left(\mathbb{R}^{n}, d_{\rho}\right)$ got by adding the single point $\infty$. Note that $\overline{\mathbb{R}^{n}}$ is a compact metric space of diameter $\pi$. Secondly, if $d$ is the Euclidean (or spherical) metric on an open set in $\mathbb{R}^{n}$ (or $\overline{\mathbb{R}^{n}}$ ), then the associated metric $l$ is called the inner Euclidean (or inner spherical) metric.

Suppose $(X, d)$ is an incomplete rectifiably connected metric space whose metric completion is denoted $(\bar{X}, \bar{d})$. We define the boundary distance $d_{X}: X \rightarrow(0, \infty)$ by the equation $d_{X}(x)=\bar{d}(x, \partial X)$, where the boundary of $X, \partial X$, is simply $\bar{X} \backslash X$. We then define the associated quasihyperbolic metric to be $d_{\rho}$ for $\rho(x)=1 / d_{X}(x)$. We sometimes write $d(x)$ in place of $d_{X}(x)$, and we normally denote the quasihyperbolic metric by $k$. We denote open balls in $(X, d)$ by $B(x, r)$ or $B_{d}(x, r)$, and closed balls by $\bar{B}(x, r)$ or $\bar{B}_{d}(x, r)$.

For the rest of this section, we assume that $(X, d)$ is incomplete and rectifiably connected, and that the associated space $(X, k)$ is geodesic. These conditions are quite mild, and certainly true when $d$ is the (inner) Euclidean or (inner) spherical metric on a Euclidean domain. More generally, the geodesic condition follows from the other two if additionally $(X, d)$ is a locally compact length space, as proved in [BoHeKo, Proposition 2.8]. We denote by $[x, y]$ any quasihyperbolic geodesic from $x$ to $y$; under the above conditions on $d$, these exist, but are not necessarily unique. It is convenient to use the same notation for paths and their images, allowing us to write for instance $w \in[x, y]$ to mean that $w$ is a point on a geodesic path from $x$ to $y$.

The following definitions come together with a parameter $C$ which is also assumed to be at least 1; this lower bound is also implicitly assumed whenever these conditions are used later.

Our first definition is that of Gromov hyperbolicity. It says that large triangles in the quasihyperbolic metric are thin. More formally we say that $(X, d)$ is $C$-kG-hyperbolic if the associated space $(X, k)$ is $C$-Gromov 
hyperbolic, meaning that

$$
\begin{array}{r}
\forall x, y, z \in X \forall[x, y],[x, z],[y, z] \forall w \in[x, y]: \\
k(w,[x, z] \cup[z, y]) \leq C .
\end{array}
$$

Note that this condition, which says that an arbitrary point on one side of a geodesic triangle is at most a bounded distance away from some point on the other two sides, amounts to a type of negative curvature assumption for the space $(X, k)$.

The notion of Gromov hyperbolicity, which makes sense in general metric spaces, was conceived in the setting of geometric group theory [Gr1], [Gr2], [GhHa]. For our case of the quasihyperbolic metric this property was extensively studied in [BoHeKo]. It turns out that the kG-hyperbolicity condition has nice consequences for the geometry of the metric itself. According to the results of this paper one such consequence, is the following "slice condition".

Given $x, y \in X$ and an arc $\gamma \in \Gamma(x, y)$, we write $(x, y ; \gamma) \in \operatorname{slice}(C)$ if there exist pairwise disjoint open subsets $\left\{S_{i}\right\}_{i=0}^{m}$ of $X, m \geq 0$, with $d_{i} \equiv \operatorname{dia}_{d}\left(S_{i}\right)<\infty$ such that

$$
\begin{aligned}
& \forall 0<i<m, \quad \forall \lambda \in \Gamma(x, y): \quad \operatorname{len}_{d}\left(\lambda \cap S_{i}\right) \geq d_{i} / C ; \\
& \forall 0 \leq i \leq m: \quad \operatorname{dia}_{k}\left(\gamma \cap S_{i}\right) \leq C ; \\
& \operatorname{len}_{d}\left(\gamma \backslash \bigcup_{i=0}^{m} S_{i}\right)=0 ; \\
& B_{d}(x, d(x) / C) \subset S_{0}, \quad B_{d}(y, d(y) / C) \subset S_{m} .
\end{aligned}
$$

We say that $(X, d)$ is a $C$-slice space, or that $X$ satisfies (Sli), if $(x, y ; \gamma) \in$ slice $(C)$ for all $x, y \in X$ satisfying $k(x, y) \geq \log 2$, and all quasihyperbolic geodesics $\gamma \in \Gamma(x, y)$.

The above slice condition is a variant of a condition introduced by Buckley and Koskela [BuKo2]; other variants of which were later used in [BuOS], [BuSt1] and [BuSt2]. In these papers, it was used in particular to obtain a variety of Sobolev- and Trudinger-type imbedding results, mostly in Euclidean domains. We shall prove in Sect. 4 that for domains on the Riemann sphere, kG-hyperbolicity implies the slice condition and all these other variant slice conditions.

The next property has its origin $[\mathrm{GeHa}]$ in the classical complex analysis. However the definition makes sense in general metric spaces. It says that geodesics in quasihyperbolic metric are quasi-minimizing the length in the original underlying metric. More formally, we say that $(X, d)$ satisfies the C-Gehring-Hayman condition if

$$
\forall x, y \in X \quad \forall[x, y]: \quad \operatorname{len}_{d}([x, y]) \leq C l(x, y) .
$$

It turns out that the Gehring-Hayman condition by itself is not enough to encode all information contained in Gromov hyperbolicity or slice conditions. 
In addition we need the following separation type condition introduced in $[\mathrm{BoHeKo}]$.

We say that $(X, d)$ satisfies a $C$-ball-separation condition if

$$
\begin{aligned}
\forall x, y \in X \forall[x, y] \forall w \in[x, y] & \forall \lambda \in \Gamma(x, y): \\
\lambda & \cap B_{d}(w, C d(w)) \neq \emptyset .
\end{aligned}
$$

The condition says that all paths from $x$ to $y$ are forced to intersect the ball $B_{d}(w, C d(w))$. (We use the term "ball-separation" to distinguish this from a later condition where such paths are forced to intersect a set that in general might not be a ball, but has certain additional useful properties.)

We are now ready to state our main theorem for domains in $\overline{\mathbb{R}^{n}}$. Note that we could replace the inner spherical metric by the more familiar inner Euclidean metric if $X$ is bounded; the constants would then also depend on $\operatorname{dia}(G)$.

Theorem 0.1. If $X \subsetneq \overline{\mathbb{R}^{n}}$ is a domain, and $d$ is the inner spherical metric, then the following conditions are equivalent.

(1) $(X, d)$ is $C_{1}-k G$-hyperbolic.

(2) $(X, d)$ is a $C_{2}$-slice space.

(3) $(X, d)$ satisfies the $C_{3}$-Gehring-Hayman and the $C_{4}$-ball separation condition.

The various constants $C_{i}$ depend only on each other and on $n$.

Note that conditions (1)-(3) are all rather general even in the Euclidean context. In particular, we claim that they all hold for all bounded simplyconnected planar domains and, more generally, whenever $X$ is a quasiconformal image of an inner uniform domain. This fact gives a large class of examples for Theorem 0.1 and was one of the initial motivations for our investigations. Let us briefly recollect the results that imply this claim: for (1) it follows by results in [BoHeKo] (specifically Theorem 1.11 and the remarks following it). In the case of (2), the claim for a slightly different slice condition follows from [BuSt2, Theorem 3.1]; for our slice condition, see Sect. 4. Finally, the claim for (3) follows from the claim for (1) together with the fact that (1) implies (3), which is proven in [BoHeKo, Sect. 7].

Moreover, Theorem 0.1 is merely an easy-to-state special case of what we actually prove in the remainder of the paper. We establish the individual implications mentioned above for varying classes of metric spaces, which include both the inner spherical case in each instance. In fact, we shall see that (1)-(3) are equivalent whenever $(X, d)$ is an incomplete locally compact length space space satisfying the following two conditions:

(a) $(X, d)$ can be equipped with a Borel measure $\mu$ which makes it into an upper regular, locally regular, locally Loewner space;

(b) The associated space $(X, k)$ is roughly starlike. 
The first condition is always true in the inner spherical or inner Euclidean cases, with $\mu$ being Lebesgue measure. The second holds for inner spherical domains if the quasihyperbolic metric is Gromov hyperbolic. In Sect. 4, we shall discuss more general situations in which conditions (a) and (b) hold. In particular we prove that rough starlikeness of $(X, k)$ holds in the presence of Gromov hyperbolicity in most instances.

Our plan of attack is as follows. After some preliminary material in the next section, we show that (2) implies (3) in Sect. 2, and also that (3) implies a variant condition, which we call (3a), consisting of Gehring-Hayman and a more useful type of separation condition than ball-separation. We discuss rough starlikeness of $(X, k)$ in Sect. 3, and prove that (1) implies (2) in Sect. 4. In Sects. 5 and 6 we show that (3a) implies (1). Finally, we develop some fundamental results related to Loewner spaces in the appendix.

Before ending this section, let us comment briefly on the various implications in Theorem 0.1. One of the motivations for this research is the paper of Bonk, Heinonen, and Koskela [BoHeKo], where it is shown that (1) implies (3), and conjectured that (a formally stronger, but actually equivalent, variant of) condition (3) implies (1). Our theorem includes a different proof of the first implication, as well as a proof of this conjecture. Finally, we remark that (3) is potentially much easier to verify than the seemingly more complicated conditions (1) and (2).

We would like to thank the referee for carefully reading the paper and for numerous suggestions that were very helpful when making final revisions. In particular, the referee spotted a gap in our original proof of Lemma 6.6.

\section{Notation and terminology}

We gather here a list of some additional notation and terminology that is used throughout the rest of the paper.

If a metric space $(X, d)$ is incomplete, rectifiably connected, and locally compact, and if the identity map $(X, d) \rightarrow(X, l)$ is continuous (as is certainly true if $(X, d)$ is a length space), then we say that $(X, d)$ is minimally nice. The significance of minimal niceness is that it guarantees that $(X, k)$ is complete, proper, geodesic, and homeomorphic to $(X, d)$; see [BoHeKo, Proposition 2.8]. Recall that a proper metric space is one in which all closed balls are compact.

Many of the results in this paper require at least that $(X, d)$ is minimally nice, but let us merely assume for the remainder of this section that $(X, d)$ is an incomplete and rectifiably connected, and that the associated space $(X, k)$ is geodesic.

If $\gamma_{1} \in \Gamma(x, y ; X)$ and $\gamma_{2} \in \Gamma(y, z ; X)$, for some $x, y, z \in X$, then $\gamma_{1}+\gamma_{2}$ denotes the joined path which first traverses $\gamma_{1}$, and then $\gamma_{2}$. More generally, $\sum_{i=0}^{j} \gamma_{j}$ is a joined path which traverses the paths $\gamma_{i}$ in their natural order. Similarly, $-\gamma$ is the reverse parametrization of $\gamma$, and $\gamma_{1}-\gamma_{2}$ is $\gamma_{1}+\left(-\gamma_{2}\right)$. 
When we say that a point $x$ lies on a path $\gamma$, we have in mind that $x=\gamma(s)$ for some specific value of $s$, which is assumed to be the same wherever $x$ is used. This allows us to use $\gamma[x, y]$ to denote the segment $\left.\gamma\right|_{[s, t]}$ of the (possibly non-injective path $\gamma$, whenever $x=\gamma(s)$ and $y=\gamma(t)$ for some $s<t$; recall that $[x, y]$ by itself denotes an unspecified quasihyperbolic geodesic segment. We use other standard interval notation analogously: for instance, $(x, y)=[x, y] \backslash\{x, y\}$. Within a proof, all instances of $[x, y]$ refer to the same quasihyperbolic geodesic path. Furthermore, whenever we select points $u, v \in[x, y]$, then $[u, v]$ always denotes the obvious subgeodesics of $[x, y]$ or $[y, x]$ (rather than an arbitrary geodesic between these points). We write $[a, b] \subset[c, d]$ if $[a, b]$ is a subgeodesic of $[c, d]$ that preserves orientation. Given $C \geq 1$, we say that a path $\gamma$ is a $\left(C, C^{\prime}\right)$-rough quasigeodesic if $\operatorname{len}_{k}(\gamma[x, y]) \leq C k(x, y)+C^{\prime}$ whenever $x, y$ lie on $\gamma$. If we assume that $C^{\prime}=0$, we speak simply of a $C$-quasigeodesic. For the rest of this paragraph, let us use $*$-geodesic to indicate any one of "geodesic", "quasigeodesic", and "rough quasigeodesic". Suppose $\gamma$ is a $*$-geodesic, and that $\alpha$ is a reparametrization of $\gamma$ with the property that $\operatorname{len}_{k}(\alpha[s, t])=t-s$ for any numbers $s<t, s, t \in I$, where $I$ denotes the domain of $\alpha$. Then $\gamma$ is $*$-geodesic segment if $I$ is of finite length, $\gamma$ is a $*$-geodesic ray if $I$ has the form $[t, \infty)$ for some $t \in \mathbb{R}$, and $\gamma$ is a $*$-geodesic line if $I=\mathbb{R}$. Note that, although we sometimes use the qualifier "quasihyperbolic" for emphasis, terms of the form " $*$-geodesic" refer by default to the quasihyperbolic metric in the rest of this paper; if more than one such metric is being used, we use notation such as a $(C ; k)$-geodesic to specify which one we mean.

We denote the maximum and minimum of a pair of numbers $s, t$ by $s \vee t$ and $s \wedge t$ respectively. Finally we note that we frequently drop constants and other parameters in many pieces of notation whenever they are unimportant or understood. For instance we may simply talk about kG-hyperbolic and slice spaces.

\section{From slice to Gehring-Hayman and separation}

In this section, we prove that the slice condition (Sli) implies the GehringHayman condition (GH) and ball-separation condition (BS). We also prove that $(\mathrm{GH})$ and $(\mathrm{BS})$ together imply another more useful separation condition (Sep). We begin with some necessary definitions.

Given $K \geq 1$, we say that a metric space $(X, d)$ lies in the class $Q C X(K)$ if it satisfies the following $K$-quasiconvexity condition:

$$
\forall x \in X, \quad \forall 0<r, \quad \forall a, b \in \bar{B}_{d}(x, r / 2): \quad l(a, b)<K r .
$$

Here as usual, $l$ denotes the arclength metric associated with $d$. More generally, we say that $(X, d)$ lies in the class $Q C X_{\mathrm{loc}}(K)$ if it satisfies the local $K$-quasiconvexity condition

$$
\forall x \in X, \quad \forall 0<r<d(x) / K, \quad \forall a, b \in \bar{B}_{d}(x, r / 2): \quad l(a, b)<K r .
$$


Of course length spaces are trivially 1-quasiconvex, and proper subdomains of $\mathbb{R}^{n}$ or $\overline{\mathbb{R}^{n}}$ with respect to the inner Euclidean or inner spherical metrics, respectively, are trivially in $Q C X(1)$. Similarly proper subdomains of $\mathbb{R}^{n}$ or $\overline{\mathbb{R}^{n}}$ with respect to the Euclidean or spherical metrics, respectively, lie in $Q C X_{\text {loc }}(1)$. The last example is a special case of the easily proven fact that if $(X, d)$ is a minimally nice subspace of a length space $\left(Y, d^{\prime}\right)$, and $X$ is open in $Y$, then $(X, d)$ lies in $Q C X_{\text {loc }}(1)$.

We now state the first main theorem of this section.

Theorem 2.3. Suppose that $(X, d) \in Q C X_{l o c}(K)$ is minimally nice and satisfies a $C$-slice condition. Then $(X, d)$ satisfies a $C^{\prime}$-Gehring-Hayman condition and $a C^{\prime}$-ball separation condition, for some $C^{\prime}=C^{\prime}(C, K)$.

When we prove that Gehring-Hayman plus separation implies Gromovhyperbolicity, it suits us to use a different type of separation condition than ball-separation. We now define this other separation condition and later show that it is implied by Gehring-Hayman plus ball-separation.

Suppose $C \geq 1$. We say that $(X, d)$ satisfies a $C$-separation condition, or simply that it satisfies condition (Sep), if for all $x, y \in X$ with $k(x, y) \geq$ $\log 2$, every quasihyperbolic geodesic $[x, y]$, and every $w \in[x, y]$, there exists an open set $S_{w} \equiv S_{w}^{[x, y]} \subset X$ such that:

$$
\begin{array}{ll} 
& w \in S_{w} \subset B_{d}(w, C d(w)) ; \\
\forall \lambda \in \Gamma(x, y): \quad \operatorname{len}_{d}\left(\lambda \cap S_{w}\right) \geq d(w) / C ; \\
& \operatorname{dia}_{k}\left([x, y] \cap S_{w}\right) \leq \log (C+1) .
\end{array}
$$

We also define $S_{w}=B(x, d(x) / 2)$ in the case $k(x, y)<\log 2$. In this case, $\left(\mathrm{Sep}_{1}\right)$ and $\left(\mathrm{Sep}_{3}\right)$ are still valid with $C=1$, and $w \in S_{w}$, but of course $\left(\mathrm{Sep}_{2}\right)$ may fail.

Theorem 2.4. Suppose that a minimally nice metric space $(X, d)$ satisfies a $C$-ball separation condition and that $(X, d)$ satisfies a $C^{\prime}$-Gehring-Hayman condition. Then $(X, d)$ also satisfies a $C_{1}$-separation condition, for some $C_{1}=C_{1}\left(C, C^{\prime}\right)$.

Our first aim is to prove the Gehring-Hayman part of Theorem 2.3. The following lemma is crucial in the proof and it will also be used later on.

Lemma 2.5. Suppose that $(X, d)$ is an incomplete rectifiably connected space. Let $u, v$ be points on a quasihyperbolic geodesic $[z, w]$ between distinct points $z, w \in X$, let $S$ be a positive length subset of $[z, w]$, and let $c \equiv k(z, w)$. Then

$$
\begin{aligned}
v & \in \bar{B}_{d}\left(u,\left(e^{c}-1\right) d(u)\right) ; \\
\left(1-e^{-c}\right) d(u) & \leq \operatorname{len}_{d}([z, w]) \leq\left(e^{c}-1\right) d(u) ; \\
e^{-c} d(u) & \leq d(v) \leq e^{c} d(u) ; \\
e^{-c} d(u) & \leq \frac{\operatorname{len}_{d}(S)}{\operatorname{len}_{k}(S)} \leq e^{c} d(u) .
\end{aligned}
$$


Proof. Let $\lambda:[0, L] \rightarrow X$ be the parametrization of $[z, w]$ by $d$-arclength. Then $d(\lambda(t)) \leq d(z)+t$, and so

$$
\operatorname{len}_{k}(\lambda)=c \geq \int_{0}^{L} \frac{d t}{d(z)+t}=\log (1+L / d(z)),
$$

thus giving the second inequality in (2.7) in the case $u=z$. The first inequality in (2.7) for $u=z$ follows in a similar manner as the second by instead using the estimate $d(\lambda(t)) \geq d(z)-t$. These inequalities for general $u$ follow by similar estimates applied separately to $[u, w]$ and $[u, z]$, as the reader can readily verify. The containment (2.6) is an immediate corollary of the second inequality in (2.7), as is the second inequality in (2.8); the first inequality then follows by symmetry. Finally, (2.8) implies (2.9).

Theorem 2.10. Suppose that $(X, d) \in Q C X_{\text {loc }}(K)$ is minimally nice and satisfies a $C$-slice condition. Then $(X, d)$ satisfies a $C^{\prime}$-Gehring-Hayman condition for some $C^{\prime}=C^{\prime}(C, K)$.

Proof. Let us fix points $x \neq y \in X$, and a quasihyperbolic geodesic $[x, y]$ and write $L=\operatorname{len}_{d}([x, y])$. Without loss of generality, we assume that $d(y) \leq d(x)$. Suppose first that $k(x, y)<\log 2$. Using the elementary estimate $e^{t}-1 \leq 2 t$ for all $0 \leq t \leq \log 2$, and the second inequality in (2.7), we see that $L \leq 2 k(x, y) d(y)$. Suppose $\lambda \in \Gamma(x, y)$ is a competing path, with $L^{\prime}=\operatorname{len}_{d}(\lambda)$. If $L^{\prime} \geq d(y) / 2$, we get a $(4 \log 2)$-GehringHayman inequality for $x, y$. If $L^{\prime}<d(y) / 2$, then $\lambda \subset B_{d}(y, d(y) / 2)$, and so $L^{\prime} \geq \operatorname{len}_{k}(\lambda) d(x) / 2 \geq k(x, y) d(x) / 2$. Putting this together with the upper bound for $L$, we get a 4-Gehring-Hayman inequality for $x, y$. We may therefore suppose that $k(x, y) \geq \log 2$.

Suppose that $d(x, y)<d(x) / 4 K$, and so $l(x, y)<d(x) / 4$. Taking $\lambda \in \Gamma(x, y)$ so that $\operatorname{len}_{d}(\lambda)<d(x) / 4$, we see that $d(u)>3 d(x) / 4$ for all $u \in \lambda$, and so $\operatorname{len}_{k}(\lambda) \leq 4 l(x, y) / 3 d(x)<1 / 3$. This contradicts the fact that $k(x, y) \geq \log 2>1 / 3$. Thus $d(x, y) \geq d(x) / 4 K$.

Let $\left\{S_{i}, d_{i}\right\}_{i=0}^{m}$ be the slices and slice diameters associated with $x, y,[x, y]$. Writing $L_{i}=\operatorname{len}_{d}\left([x, y] \cap \bar{S}_{i}\right)$, we get upper bounds for the numbers $L_{i}$ in the case of small and large slices by separate arguments. We first consider small slices, meaning slices $S_{i}$ for which $d_{i}<d\left(z_{i}\right) / 3 K$ for some point $z_{i} \in[x, y] \cap S_{i}$. Fixing one such small slice $S_{i}$, we let $a, b$ be the first and last points of intersection of $[x, y]$ with $\bar{S}_{i}$, and let $\lambda \in \Gamma(a, b)$ be such that $\operatorname{len}_{d}(\lambda)<K d(a, b)$. Thus $\operatorname{len}_{d}(\lambda)<d\left(z_{i}\right) / 3$, and so $d(w)>$ $d\left(z_{i}\right)[1-1 /(3 K)-1 / 3] \geq d\left(z_{i}\right) / 3$ for all points $w$ on $\lambda$. Thus

$$
\operatorname{len}_{k}[a, b]<\frac{3 \operatorname{len}_{d}(\lambda)}{d\left(z_{i}\right)}<\frac{3 K d_{i}}{d\left(z_{i}\right)} .
$$

The rightmost quantity in (2.11) is less than 1 , so (2.8) implies that $d(w)<$ $e d\left(z_{i}\right)$ for all $w \in[a, b] \cap \bar{S}_{i}$. Thus

$$
L_{i} \leq \operatorname{len}_{d}[a, b] \leq e d\left(z_{i}\right) \operatorname{len}_{k}[a, b] \leq 3 e K d_{i} .
$$


We next turn to the big slices, i.e., slices $S_{i}$ such that $d_{i} \geq d(z) / 3 K$ for all $z \in[x, y] \cap S_{i}$. Consequently, from $\left(\mathrm{Sli}_{2}\right)$ we see that for all $0<i<m$,

$$
L_{i} \leq 3 K d_{i} \operatorname{len}_{k}\left(\gamma \cap S_{i}\right) \leq 3 C K d_{i} .
$$

As for the case $i=0$, the quasihyperbolic length of the segment of $[x, y]$ from $x$ to its last intersection point with $\overline{S_{0}}$ is at most $C$ according to $\left(\mathrm{Sli}_{2}\right)$. Using (2.7) from Lemma 2.5, we deduce that $L_{0} \leq(\exp (C)-1) d(x)$. Similarly $L_{m} \leq(\exp (C)-1) d(y)$. Combining these last two estimates with (2.12), (2.13), and $\left(\mathrm{Sli}_{3}\right)$, we deduce that

$$
L \leq C_{1}\left(d(x)+d(y)+\sum_{i=1}^{m-1} d_{i}\right),
$$

where $C_{1}=(\exp (C)-1) \vee(3(e \vee C) K)$. On the other hand, $\left(\mathrm{Sli}_{1}\right)$ implies that

$$
\forall \lambda \in \Gamma(x, y), 0<i<m: \operatorname{len}_{d}\left(\lambda \cap S_{i}\right) \geq d_{i} / C,
$$

while $\left(\mathrm{Sli}_{4}\right)$ and the fact that $d(x, y)>d(x) / 4 K$ together imply that

$$
\begin{aligned}
& \operatorname{len}_{d}\left(\lambda \cap S_{0}\right) \geq d(x) /(4 K \vee C), \\
& \operatorname{len}_{d}\left(\lambda \cap S_{m}\right) \geq d(y) /(4 K \vee C) .
\end{aligned}
$$

Thus

$$
\begin{aligned}
\forall \lambda \in \Gamma(x, y): \operatorname{len}_{d}(\lambda) & \geq \sum_{i=0}^{m} \operatorname{len}_{d}\left(\lambda \cap S_{i}\right) \geq \\
& \geq C_{2}^{-1}\left(d(x)+d(y)+\sum_{i=1}^{m-1} d_{i}\right),
\end{aligned}
$$

where $C_{2}=8 K \vee 2 C$. Combining this inequality with (2.14), we deduce a $C^{\prime}$-Gehring-Hayman condition with $C^{\prime}=C_{1} C_{2}$.

Theorem 2.10 proves one part of Theorem 2.3. The other part follows from the following theorem.

Theorem 2.15. Suppose $(X, d)$ is a minimally nice metric space that satisfies a $C$-slice condition. Then $(X, d)$ satisfies a $C_{1}$-ball separation condition, where $C_{1}=2 \vee\left(C^{2} \exp (C)\right)$.

Proof. Fix two distinct points $x, y \in X$, and let $\gamma=[x, y]:[0, L] \rightarrow X$ be a geodesic for $x, y$, and let $w=\gamma(t)$ be a point on $\gamma$. Without loss of generality, we assume that $d(y) \leq d(x)$. If $k(x, y)<\log 2$ and $u \in[x, y]$, then by (2.6), we have $x, y \in B_{d}(u, 2 d(u))$, as desired. We may suppose therefore that $k(x, y) \geq \log 2$. 
We fix a point $w=\gamma(t)$, and let $\left\{S_{i}, d_{i}\right\}_{i=0}^{m}$ be the slices and slice diameters associated with $x, y,[x, y]$. Since there are only finitely many slices $S_{i}$, we can choose an index $j$ and numbers $t_{n} \in[0, L]$ such that $\lim _{n \rightarrow \infty} t_{n}=t$ and $w_{n}=\gamma\left(t_{n}\right) \in S_{j}$.

Let $\gamma_{j} \equiv \gamma \cap S_{j}$. By $\left(\mathrm{Sli}_{2}\right)$ and (2.9), it readily follows that

$$
\operatorname{len}_{d}\left(\gamma_{j}\right) / d(z) \leq C \exp (C), \quad z \in \gamma_{j} .
$$

Combining this with $\left(\mathrm{Sli}_{1}\right)$, we deduce that if $0<j<m$, then $d_{j} \leq$ $C^{2} \exp (C) d(z)$. Taking $z=w_{n}$ and passing to a limit, we see that $d_{j} \leq$ $C_{1} d(w)$. Thus $\overline{S_{j}} \subset B\left(w, C_{1} d(w)\right)$, and the $C_{1}$-ball separation condition for the data $\{x, y,[x, y], w\}$ follows from $\left(\operatorname{Sli}_{1}\right)$. The endpoint cases $j \in\{0, m\}$ are even easier since then $\gamma_{j}$ must include either $x$ or $y$, and so a $\left(C_{1} / C^{2}\right)$-ball separation condition follows for the data.

Proof of Theorem 2.4. To prove the first statement suppose we are given separation data $x, y,[x, y]$, and $w \in[x, y]$, with $k(x, y)>\log 2$. It follows from (2.7) that $\operatorname{len}_{d}([x, y]) \geq d(w) / 2$. Now if we were to choose $S_{w}$ to be $B_{d}\left(w, 2 C C^{\prime} d(w)\right)$, then $\left(\operatorname{Sep}_{1}\right)$ and $\left(\operatorname{Sep}_{2}\right)$ would readily follow, but $\left(\mathrm{Sep}_{3}\right)$ would typically fail.

We instead define the separation set $S_{w}$ by

$$
S_{w}=B_{d}\left(w, 4 C C^{\prime} d(w)\right) \backslash\left(\left(X \backslash B_{k}(w, \log 2)\right) \cap[x, y]\right) .
$$

We claim that $S_{w}$ satisfies the $C_{1}$-separation condition, where $C_{1}=4 C C^{\prime}$. Clearly $\left(\mathrm{Sep}_{1}\right)$ holds, and $\left(\mathrm{Sep}_{3}\right)$ is just as obvious once we note that $\log \left(C_{1}+1\right)>2 \log 2$. However the fact that $S_{w}$ satisfies $\left(\mathrm{Sep}_{2}\right)$ is far from obvious, so let us fix an arbitrary path $\lambda \in \Gamma(x, y)$, and suppose for the sake of contradiction that $\operatorname{len}_{d}(A)<d(w) / C_{1}$, where $A=\lambda \cap S_{w}$. We may as well assume that $\lambda:[0, L] \rightarrow X$ is parametrized by arclength.

We define $w_{-}$to be the point in $[x, w]$ with $k\left(w_{-}, w\right)=(\log 2) \wedge k(x, w)$, and $w_{+}$to be the point in $[w, y]$ with $k\left(w_{+}, w\right)=(\log 2) \wedge k(w, y)$, so that the part of $[x, y]$ that lies in $S_{w}$ is $\left(w_{-}, w_{+}\right)$plus perhaps one or both endpoints of this geodesic segment (an endpoint $e$ is included precisely when $k(e, w)<\log 2$, and so $e \in\{x, y\})$.

Since $k\left(w_{-}, w_{+}\right) \geq \log 2, \operatorname{len}_{d}\left(\left[w_{-}, w_{+}\right]\right) \geq d(w) / 2$. With this notation, $A$ is the set of points in $\lambda \cap B_{d}\left(w, 4 C C^{\prime} d(w)\right)$ that lie in neither $\left[x, w_{-}\right]$nor $\left[w_{+}, y\right]$.

Also let $t_{-} \in[0, L)$ be defined by the equation

$$
t_{-}=\sup \left\{t \in[0, L] \mid \lambda(t) \in\left[x, w_{-}\right]\right\},
$$

let $t_{+} \in\left[t_{-}, L\right]$ be defined by

$$
t_{+}=\inf \left\{t \in\left[t_{-}, L\right] \mid \lambda(t) \in\left[w_{+}, y\right]\right\},
$$

so that $v_{-} \equiv \lambda\left(t_{-}\right) \in\left[x, w_{-}\right], v_{+} \equiv \lambda\left(t_{+}\right) \in\left[w_{+}, y\right]$. It is clear that

$$
\left\{\lambda(t): t \in\left(t_{-}, t_{+}\right)\right\} \cap B_{d}\left(w, 4 C C^{\prime} d(w)\right) \subseteq A .
$$


Set $\tilde{\lambda}:=\left\{\lambda(t): t \in\left(t_{-}, t_{+}\right)\right.$. Suppose first that $\tilde{\lambda} \subseteq B_{d}\left(w, 4 C C^{\prime} d(w)\right)$. In this case $\tilde{\lambda} \subseteq A$. Gehring-Hayman implies that

$$
\begin{aligned}
& \operatorname{len}_{d} \tilde{\lambda}=t_{+}-t_{-} \geq \frac{1}{C^{\prime}} \operatorname{len}_{d}\left(\left[\lambda\left(t_{-}\right), \lambda\left(t_{+}\right)\right]\right) \geq \\
& \geq \frac{1}{C^{\prime}} \operatorname{len}_{d}\left(\left[w_{-}, w_{+}\right]\right) \geq \frac{d(w)}{2 C^{\prime}}>d(w) / C_{1} .
\end{aligned}
$$

This leads to a contradiction with $\operatorname{len}_{d}(A)<d(w) / C_{1}$.

In the second case, when $\tilde{\lambda}$ has a point outside of $B_{d}\left(w, 4 C C^{\prime} d(w)\right)$ we apply (BS) for the data $\left\{v_{-}, v_{+}, w \in\left[v_{-}, v_{+}\right]\right\}$. This implies that $\tilde{\lambda}$ must intersect the smaller ball $B_{d}(w, C d(w))$ and so $\tilde{\lambda}$ has a subarc $\beta \subseteq$ $B_{d}\left(w, 4 C C^{\prime} d(w)\right)$ such that $\operatorname{len}_{d} \beta \geq\left(4 C C^{\prime}-C\right) d(w)$. Since $\beta \subseteq A$ we obtain again a contradiction to $\operatorname{len}_{d}(A)<d(w) / C_{1}$.

One might wonder if there is an implication one way or the other between the Gehring-Hayman and ball-separation conditions, at least in the setting of bounded Euclidean domains. We do not know whether ball-separation (or separation) implies Gehring-Hayman, but we now give an example of a quasiconvex planar domain $G$ for which the (inner) Euclidean metric satisfies a Gehring-Hayman condition, but not a ball-separation condition. To construct $G$, we "weld" the square $(-1,1)^{2}$ to the sequence of squares $Q_{j}=\left(a_{j}, a_{j}+l_{j}\right) \times\left(1,1+l_{j}\right)$, where $a_{j}=2^{-j}$, and $l_{j}=4^{-j}, j \in \mathbb{N}$, and the welding process consists of adding all points on the interval whose coordinates are of the form $(x, 1)$ for some $a_{j}<x<a_{j}+l_{j}$, except for all points of the form $\left(a_{j}+i l_{j} / j, 1\right), i \in \mathbb{N}, i<j$. Taking $z_{j}$ to be the center of $Q_{j}$, the large number of passages forces any $C$-ball-separation condition to fail in the vicinity of the $j$ th weld for any geodesic from the origin to $z_{j}$ whenever $j \geq j_{0}=j_{0}(C)$. A case analysis shows that $(G, d)$ satisfies a Gehring-Hayman condition.

Let us finish this section by commenting on a minor difference between the ball-separation condition we are using and the separation condition defined at the end of Sect. 7 of [BoHeKo]. The latter is the formally stronger condition

$$
\begin{array}{r}
\forall x, y \in X \quad \forall[x, y] \forall w \in[x, y], \\
u \in[x, w), v \in(w, y] \forall \lambda \in \Gamma(u, v): \\
\lambda \cap B_{d}(w, C d(w)) \neq \emptyset .
\end{array}
$$

In fact these conditions are equivalent. To see this, suppose that $(X, d)$ satisfies (BS) and let $x, y, w, u, v, \lambda$ be as in (SBS). Since the $\operatorname{arc}[u, v] \subseteq$ $[x, y]$ connecting $u, v \in X$ is itself a geodesic, we can use (BS) with data $\{u, v, w \in[u, v]\}$ to deduce that $\lambda \cap B_{d}(w, C d(w)) \neq \emptyset$, as required.

\section{Gromov hyperbolicity implies rough starlikeness}

In the next section we prove that, in a general class of metric spaces, kG-hyperbolicity implies a slice condition. This class of spaces include 
bounded domains in complete metric measure spaces that are $Q$-regular and $Q$-Loewner. This includes a large variety of non-Euclidean spaces such as Carnot groups, sub-Riemannian and Riemannian manifolds which all satisfy the Loewner condition. We refer to [HeKo] for a detailed exposition on analysis in Loewner spaces. We recall the definition and basic properties in the Appendix.

In proving that Gromov hyperbolicity implies a slice condition the property that $(X, k)$ is roughly starlike also plays a crucial role. This property has not been treated in as much detail as the Loewner property. Therefore we pause and devote this section to this condition. We show that rough starlikeness follows essentially from kG-hyperbolicity in a rather general setting.

It is shown in Lemma 7.8 of [BoHeKo] that if a proper subdomain $X$ of $\overline{\mathbb{R}^{n}}$ is kG-hyperbolic with respect to the inner spherical metric, then $(X, k)$ is roughly starlike with respect to any basepoint $w$ that maximizes the spherical distance to $\partial X$. In this section, our main theorem generalizes that result, in the process providing a large class of spaces in which this implication is valid, although we delay until the end of the next section a discussion of what spaces are covered by the theorem.

If $(X, d)$ is a minimally nice metric space, then $(X, k)$ is said to be $K$ roughly starlike, $K \geq 0$, if there exists a basepoint $w$ such that every point in the space is within a distance $K$ of some geodesic ray emanating from $w$.

Throughout the rest of this section, we define $A\left(z, r, C, C^{\prime}\right)$ to be the annulus $\bar{B}_{\bar{d}}(z, C r) \backslash B_{\bar{d}}\left(z, r / C^{\prime}\right)$, whenever $z \in \bar{X}, r>0, C, C^{\prime} \geq 1$, and $(\bar{X}, \bar{d})$ is the metric completion of a metric space $(X, d)$. As a special case, we write $A(z, r, C)=A(z, r, C, C)$.

Given $C \geq 1$, we say that $(X, d)$ is in the class $Q L(C)$ if it is minimally nice and it satisfies the following three conditions:

(a) If $x, x^{\prime} \in X, z \in \partial X, x, x^{\prime} \in A(z, r, 1,8 C)$, and $A(z, r, 8 C) \subset X$, then there exists a path $\lambda \in \Gamma\left(x, x^{\prime} ; A\left(z, r, 8 C^{2}\right)\right)$ such that $\operatorname{len}_{d}(\lambda) \leq 2 C r$.

(b) If $x \in X, z \in \partial X, x \in A(z, r, 2)$, and $A(z, r, 4 C) \cap \partial X \neq \emptyset$, then there exists a point $y \in A(z, r, 4 C) \cap \partial X$ and a path $\eta \in \Gamma\left(x, y ; A\left(z, r, 8 C^{2}\right)\right)$ such that $\operatorname{len}_{\bar{d}}(\eta) \leq 6 C^{2} r$.

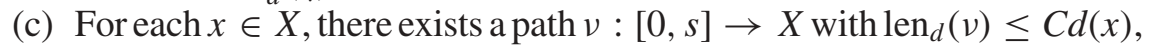
$v(0)=x$, and $d(v(t))>d(v(s))=d(x) / 2$ for all $0 \leq t<s$.

We chose the notation $Q L(C)$ because the three parts of this condition are combinations of special cases of two well-known conditions that are usually termed quasiconvexity and LLC-2. Let us also write $Q L(C)(\mathrm{a}), Q L(C)(\mathrm{b})$, $Q L(C)$ (c), to refer to the three individual conditions above.

It is straightforward to verify that (inner) Euclidean and (inner) spherical domains are in the class $Q L(1)$. It is similarly easy to prove from Remark 7.2 that a bounded, upper $Q$-regular, $Q$-Loewner minimally nice metric measure space is in $Q L(C)$, where $C$ depends quantitatively on the assumptions (see the proof of Proposition 4.19 for more details).

We are now ready to state the main theorem of this section. 
Theorem 3.1. Let $(X, d) \in Q L(C)$ be $C^{\prime}$-kG-hyperbolic and $M \equiv$ $\sup _{x \in X} d(x)<\infty$. Then there exists $K=K\left(C, C^{\prime}\right)$ such that $(X, k)$ is $K$-roughly starlike with respect to any basepoint $w$ for which $d(w)>M / 2$.

The following lemma, which actually only uses minimal niceness and part (c) of the definition of $Q L(C)$ is the main tool in our proof of Theorem 3.1.

Lemma 3.2. Suppose that $(X, d) \in Q L(C)$. Then for each $u_{0} \in X$, there exists a rough quasigeodesic ray $\gamma:[0, L) \rightarrow X$ emanating from $u_{0}$, and parametrized by d-arclength such that $d(\gamma(L-t)) \geq t / 4 C$ for all $0<t \leq L$, $\gamma(t) d$-converges to some point $z \in \partial X$ as $t \rightarrow L^{-}$, and

$$
\begin{array}{r}
\operatorname{len}_{k}(\gamma[u, v]) \leq 2 C\left[1+\log _{2}(3 C)+\left|\log _{2}(d(u) / d(v))\right|\right], \\
\text { for all points } u, v \text { lying on } \gamma .
\end{array}
$$

Proof. Suppose $u_{0} \in X$ is given. We define $\gamma$ by stringing together a sequence of paths $\gamma_{i} \in \Gamma\left(u_{i-1}, u_{i} ; X\right), i \in \mathbb{N}$, where $d\left(u_{i}\right)=2^{-i} d\left(u_{0}\right)$, $i \in \mathbb{N}$. We define $\gamma_{1}$ to be the path $v$ given by $Q L(C)$ (c) for $x=u_{0}$, and let $u_{1}=v(s)$. Inductively, for each $i \in \mathbb{N}$, let $v$ and $s$ be the data given by $Q L(C)$ (c) for $x=u_{i-1}$. We then define $\gamma_{i}:\left[0, l_{i}\right] \rightarrow X$ to be the $d$-arclength reparametrization of $v$, and $u_{i}$ to be $v(s)$; note that $l_{i} \leq 2^{-i+1} C d\left(u_{0}\right)$. Writing $L_{i}=\sum_{j=1}^{i} l_{j}, i \in \mathbb{N}$, and $L=\sum_{j=1}^{\infty} l_{j}$, we define the path $\gamma:[0, L) \rightarrow \bar{X}$ by the equations $\gamma(t)=\gamma_{i}\left(t-L_{i-1}\right)$, for all $L_{i-1} \leq t \leq L_{i}$. Then $\gamma$ is parametrized by $d$-arclength, $L \leq 4 C d\left(u_{0}\right)$, and clearly $\gamma(t) d$-approaches some $z \in \partial X$ as $t \rightarrow L^{-}$. The estimate $d(\gamma(L-t)) \geq t / 4 C$ is also clear.

Suppose $u=\gamma(s)$ and $v=\gamma(t)$, where $s<t, L_{i-1} \leq s \leq L_{i}$, and $L_{j-1} \leq t \leq L_{j}$, for some $i \leq j \in \mathbb{N}$. Since all points in $\gamma_{i}$ are of distance at least $2^{-i} d\left(u_{0}\right)$ from $\partial X$, and $l_{i} \leq 2^{-i+1} C d\left(u_{0}\right)$, it follows that len ${ }_{k}\left(\gamma_{i}\right) \leq 2 C$, and so $\operatorname{len}_{k}(\gamma[u, v]) \leq 2 C(j-i+1)$. On the other hand,

$$
2^{j-1} \frac{1}{3 C} \leq \frac{d(u)}{d(v)} \leq 2^{j-i} 3 C,
$$

and so $j-i \leq|\log (d(u) / d(v))|+\log _{2}(3 C)$. The estimate (3.3) is now clear.

We now record the following elementary but useful estimate, whose simple proof we leave to the reader.

$$
k(u, v) \geq \log \left(1+\frac{l(u, v)}{d(u) \wedge d(v)}\right) .
$$

This implies the inequality $k(u, v) \geq|\log (d(u) / d(v))|$, which together with (3.3) proves that $\gamma$ is a rough quasigeodesic. 
Let us pause to recap some basics of Gromov hyperbolicity in the context of a quasihyperbolic space $(X, k)$ which we assume to be Gromov hyperbolic, proper, and geodesic; recall that the latter two assumptions follow from minimal niceness of $(X, d)$. For proofs of the results in the next two paragraphs and much more on hyperbolicity, we refer the reader to [GhHa], [CoDePa]; see also [BoSc] and [BoHeKo].

We define the Gromov boundary of $X$, denoted $\partial_{G} X$, to be the set of all geodesic rays emanating from some fixed point $w \in X$, modulo the equivalence relation given by taking any two rays which lie within a bounded Hausdorff distance of each other as equivalent; this definition is independent of $w$. Morally, $\partial_{G} X$ consists of the "points at infinity" of $(X, k)$. It may or may not be homeomorphic to the boundary $\partial X$ defined previously. For example if $X$ is a Euclidean slit disk (e.g., the unit disk in the complex plane less the positive real axis), then $\partial_{G} X$ is not homeomorphic to $\partial X$, but rather to the unit circle; each point on the positive real axis corresponds to two points in $\partial_{G} X$.

Proof of Theorem 3.1. We assume without loss of generality that $C \geq 2$. Suppose $x \in X$. Let $\gamma:[0, L) \rightarrow X$ be a rough quasigeodesic ray emanating from $x$, as in Lemma 3.2, such that $\gamma(t) d$-converges to $z \in \partial X$. We define the path $\gamma_{1}:(0, L] \rightarrow X$ by the equation $\gamma_{1}(t)=\gamma(L-t)$, for all $0<t \leq L$. Letting $r=d(z, x)$, we certainly have $x \in A(z, r, 2)$.

Suppose $A(z, r, 2 C) \cap \partial X$ is non-empty. Let $y$ and $\eta$ be as in part (b) of the $Q L(C)$ definition. As we move along $\eta$ from $x$ to $y$, let $x^{\prime}$ be the first point with $d\left(x^{\prime}\right)=r /\left(32 C^{3}\right)$, and let $\gamma_{2}$ be the segment of $\eta$ from $x$ to $x^{\prime}$. Finally, let $\gamma_{3}:[0, L) \rightarrow X$ be a rough quasigeodesic ray from $x^{\prime}$ that converges to some point $z^{\prime} \in \partial X$ and satisfies the conclusions of Lemma 3.2.

We claim that $\gamma$ is a rough quasigeodesic line. Since $\gamma_{1}$ and $\gamma_{3}$ are rough quasigeodesic rays and

$$
\operatorname{len}_{k}\left(\gamma_{2}\right) \leq \frac{\operatorname{len}_{d}\left(\lambda_{2}\right)}{r /\left(32 C^{3}\right)} \leq 192 C^{5},
$$

it suffices to show the quasigeodesic property for a pair of points $u$ on $\gamma_{1}$ and $v$ on $\gamma_{3}$. Since $\operatorname{len}_{k}(\gamma[u, v]) \leq \operatorname{len}_{k}\left(\gamma_{1}[u, x]\right)+192 C^{5}+\operatorname{len}_{k}\left(\gamma_{3}\left[x^{\prime}, v\right]\right)$, it suffices to bound the first and last term in this sum by a constant plus $k(u, v)$.

We first consider $K_{1} \equiv \operatorname{len}_{k}\left(\gamma_{1}[u, x]\right)$. Note that

$$
d(z, v) \geq d\left(z, x^{\prime}\right)-d\left(x^{\prime}, v\right) \geq \frac{r}{4 C^{2}}-\frac{r}{8 C^{2}}=r /\left(8 C^{2}\right) .
$$

If $d(u, z)<r /\left(16 C^{2}\right)$, then $d(u, v)>r /\left(16 C^{2}\right)$ and so by (3.4) we have

$$
k(u, v) \geq \log \left(1+\frac{r /\left(16 C^{2}\right)}{d(u) \wedge d(v)}\right) .
$$


But by (3.3),

$$
\begin{aligned}
K_{1} & \leq 2 C\left(1+\log _{2}(3 C)+\left|\log \frac{d(x)}{d(u)}\right|\right) \\
& \leq 2 C\left(1+\log _{2}(3 C)+\log \frac{r}{d(u)}\right) .
\end{aligned}
$$

Putting together the last two estimates, we get the required bound for $K_{1}$. On the other hand if $d(u, z) \geq r /\left(16 C^{2}\right)$, then $d(u) / d(x) \leq 4 C$ by Lemma 3.2. Moreover $d(x) \leq d(x, z)=r$ and again by Lemma 3.2

$$
d(u) \geq \frac{\operatorname{len}_{d}(\gamma[u, z])}{4 C} \geq \frac{d(u, z)}{4 C} \geq \frac{r}{64 C^{3}} .
$$

This implies that $d(x) / d(u) \leq 64 C^{3}$. So by (3.3), we see that

$$
K_{1} \leq 2 C\left(7+\log _{2}(3 C)+3 \log _{2} C\right) .
$$

We now consider $K_{3} \equiv \operatorname{len}_{k}\left(\gamma_{3}\left[x^{\prime}, v\right]\right)$. We first use (3.3) to get the inequality

$$
K_{3} \leq 2 C\left(1+\left|\log \frac{d\left(x^{\prime}\right)}{d(v)}\right|\right) \leq 2 C\left(1+\left|\log \frac{r}{32 C^{3} d(v)}\right|\right) .
$$

If $d(u, z)<r /\left(16 C^{2}\right)$, the desired bound follows by combining (3.6) and (3.5). If instead $d(u, z) \geq r /\left(16 C^{2}\right)$, and so $r /\left(16 C^{2}\right) \geq d(u) \geq r /\left(64 C^{3}\right)$, then the desired bound follows by combining (3.6) with the elementary estimate $k(u, v) \geq|\log (d(u) / d(v))|$ and the upper bound $d(v) \leq(2 C$ $+1) d\left(x^{\prime}\right)<r /\left(8 \bar{C}^{2}\right)$.

But rough quasigeodesics lines are never far from honest geodesic lines in a Gromov hyperbolic space according to [CoDePa, Theorem 3.3.1]. So $x$ lies within a bounded quasihyperbolic distance of a geodesic line $(a, b)$ joining two points $a, b \in \partial_{G} X$. Let $\rho_{a}, \rho_{b}$ be geodesic rays from $w$ to $a$ and $b$, respectively. Now the "thin triangles" property (Hyp) is valid for arbitrary points $x, y, z \in X \cup \partial_{G} X$ and any associated geodesics, as long as we replace the hyperbolicity constant $C$ by $24 C$; see [CoDePa, 2.2.2]. Consequently $x$ lies within a bounded distance of either $\rho_{a}$ or $\rho_{b}$.

Finally, we must consider the case where there is no point $y \in \partial X$ which also lies in the annulus $A(z, r, 2 C)$. The part of $\gamma_{1}$ that is near $z$ is within a bounded Hausdorff distance of some final segment of a geodesic ray $\theta$ emanating from $w$. Note that $\theta$ must necessarily approach $z$, and that $d(w, z) \geq d(w) \geq d(x) / 2 \geq r / 8 C$. Let $x^{\prime}$ be the first point on $\theta$ such that $d\left(x^{\prime}, z\right)=r / 8 C$, and let $\lambda$ be the path given by $Q L(C)(\mathrm{a})$. Since $\operatorname{len}_{d}(\lambda)<2 C r, \lambda$ stays in the annulus $\bar{B}(z,(C+1) r) \backslash B(z, r /(8 C))$. It is now easy to deduce that $\operatorname{len}_{k}(\lambda)$ is bounded, so we are done. 
We end this section by giving an example of a quasiconvex bounded planar domain $G$ where the quasihyperbolic metric $k$ associated to the (inner) Euclidean metric is not roughly starlike (but $(G, k)$ is not Gromovhyperbolic either). We define $G=D \backslash \bigcup_{j=2}^{\infty} E_{j}$, where $D$ is the unit disk and $E_{j}$ consists of $j$ equally spaced points on the boundary of the disk $D_{j}=B\left(z_{j}, r_{j}\right)$, where $z_{j}=\left(1-2^{-j}, 0\right)$, and $r_{j}=4^{-j}$. It is not hard to see that there is some integer $j_{0}$ such that every geodesic ray emanating from the origin remains outside $D_{j}$ for all $j \geq j_{0}$. Thus the points $z_{j}$ are quasihyperbolically far from all geodesic rays. It is also interesting to note that when $j$ is large, no geodesic segment from the origin to $z_{j}$ can be continued outside of $c D_{j}$, where $c<1 / 2$ tends to zero as we let $j$ tend to infinity.

We also note that we cannot strengthen the conclusions of the main results in this section from roughly starlike to starlike (the latter meaning that geodesic rays pass through every point, as they do in any Euclidean ball). To see this, we simply modify the previous example so that every $E_{j}$ consists of exactly 1000 equally spaced points. The resulting domain is $\mathrm{kG}$-hyperbolic, but the points $z_{j}$ are still not on geodesic rays.

\section{Gromov hyperbolicity implies slice}

Our main theorem in this section is as follows. The definitions of (local) regularity, (local) Loewner, and some related lemmas have been relegated to the appendix, Sect. 7.

Theorem 4.1. Suppose $(X, d, \mu)$ is minimally nice, upper $Q$-regular, locally $Q$-regular, and locally $Q$-Loewner. Suppose also that $(X, d)$ is a length space, and that $(X, k)$ is Gromov hyperbolic and roughly starlike. Then $(X, d)$ satisfies a slice condition, quantitatively.

This theorem is applicable in particular if $(X, d, \mu)$ is a proper subdomain of $\overline{\mathbb{R}^{n}}$, with the inner spherical metric and Lebesgue measure attached. In this case, upper regularity, local regularity and the local Loewner property are clearly true and, since inner spherical domains lie in the class $Q L(1)$, Theorem 3.1 implies that $(X, k)$ is roughly starlike whenever it is Gromov hyperbolic. Thus the above theorem implies that a kG-hyperbolic inner spherical domain satisfies a slice condition. By Theorem 3.1 and the remarks that precede it, $(X, k)$ is roughly starlike whenever $(X, d, \mu)$ is a minimally nice, bounded, upper $Q$-regular, $Q$-Loewner space, and $(X, k)$ is Gromov hyperbolic. We generalize this result to domains in such spaces in Proposition 4.19. So the rough starlike assumption in Theorem 4.1 is readily available in most applications.

Let us briefly overview the contents of this section. We first introduce and discuss a variant slice condition slice ${ }^{+}$which is stronger than slice. Then we discuss some concepts that we need to prove the main theorem, including the uniform deformations $\left(X, d_{\epsilon}\right)$ of a metric space $(X, d)$ satisfying (Hyp) that 
were introduced by Bonk, Heinonen, and Koskela [BoHeKo]. The benefit of $\left(X, d_{\epsilon}\right)$ is that it is a uniform space provided that $(X, k)$ is Gromov hyperbolic. Using this, we prove that $\left(X, d_{\epsilon}\right)$ satisfies a slice ${ }^{+}$condition, and then use a conformal modulus argument to deduce that $(X, d)$ also satisfies a slice ${ }^{+}$condition. Finally, we explicitly describe a large class of spaces on which kG-hyperbolicity implies slice ${ }^{+}$.

The main reason for proving the stronger slice ${ }^{+}$condition is that it immediately implies (if we take $d$ to be the inner Euclidean metric) all of the slice-type conditions defined for a variety of purposes in [BuKo2], [BuSt1], and [BuSt2]. (The same is not true of our slice condition.) Thus it follows from the results in this section that a bounded kG-hyperbolic Euclidean domain satisfies all these slice-type conditions, and that slice ${ }^{+}$and slice are equivalent on inner spherical domains, or more generally on all length spaces that are minimally nice and satisfy conditions (a) and (b) in the discussion after Theorem 0.1.

The slice ${ }^{+}$condition involves the conformal modulus $\bmod _{Q}$, defined in Sect. 7, a concept that is central to our method of proof. Suppose $(X, d, \mu)$ is a minimally nice, upper $Q$-regular metric space, and that $0<c<1, C \geq 6$. Given $x, y \in X$ and $\gamma \in \Gamma(x, y)$, suppose there exist pairwise disjoint open subsets $\left\{S_{i}\right\}_{i=0}^{m}$ of $X, m \geq 0$, with $d_{i} \equiv \operatorname{dia}_{d}\left(S_{i}\right)<\infty$, and points $\left\{x_{i}\right\}_{i=0}^{m}$ on $\gamma$, such that $x_{i} \in S_{i}$, with $d_{i} / C \leq d\left(x_{i}\right) \leq C d_{i}$, and $x_{0}=x, x_{m}=y$. We then write $(x, y ; \gamma) \in$ slice ${ }^{+}(C, c)$ if additionally $\left(\mathrm{Sli}_{2}\right)$ and $\left(\mathrm{Sli}_{3}\right)$ both hold and, defining $E_{i} \equiv \bar{B}_{d}\left(x_{i}, c d\left(x_{i}\right)\right)$, the following two conditions also hold:

$\left(\mathrm{Sli}_{1}^{+}\right)$For $0<i<m$, and every $\lambda \in \Gamma(x, y)$, there exists a closed subpath $\lambda_{i}$ of $\lambda$ such that $\lambda_{i} \in \overline{S_{i}}$ and $\operatorname{dia}_{d}\left(\lambda_{i}\right) \geq d_{i} / C$.

$\left(\mathrm{Sli}_{4}^{+}\right)$For $0 \leq i \leq m$, the ball $6 E_{i}$ is contained in $S_{i}$.

We say that $(X, d, \mu)$ satisfies the $(C, c)$-slice ${ }^{+}$condition if $(x, y ; \gamma) \in$ slice $^{+}(C, c)$ for every $x, y \in X$ and every connecting geodesic segment $\gamma$. Clearly the $(C, c)$-slice ${ }^{+}$condition implies a $\left(C^{\prime}, 0\right)$-slice ${ }^{+}$condition, where $C^{\prime}=C \vee c^{-1}$.

We shall actually prove the following stronger version of Theorem 4.1.

Theorem 4.2. Suppose $(X, d, \mu)$ is minimally nice, upper $Q$-regular, locally $Q$-regular, and locally $Q$-Loewner. Suppose also that $(X, d)$ is a length space, and that $(X, k)$ is Gromov hyperbolic and roughly starlike. Then $(X, d)$ satisfies a slice ${ }^{+}$condition, quantitatively.

We define one more slice-type condition. Specifically, we define the mod-slice $(C, c)$ condition to be identical to the slice ${ }^{+}(C, c)$ condition, except that we replace $\left(\mathrm{Sli}_{1}^{+}\right)$by the following modulus variant:

(Sli $\left.{ }_{5}\right)$ For $0<i<m$, and every $\lambda \in \Gamma(x, y)$, there exists a closed subpath $\lambda_{i}$ of $\lambda$ such that $\lambda_{i} \in \overline{S_{i}}$ and $\bmod _{Q}\left(\lambda_{i}, E_{i}\right) \geq C^{-1}$.

The meaning of the condition " $(x, y ; \gamma) \in \bmod -\operatorname{slice}(C, c)$ " is as one would expect. 
We suspect that mod-slice may not be implied by Gromov hyperbolicity under the hypotheses of Theorem 4.1, but it is nevertheless rather useful in our proof of Theorem 4.2. It is clear that in a (global) Loewner space, $\left(\mathrm{Sli}_{1}^{+}\right)$implies $\left(\mathrm{Sli}_{5}\right)$ quantitatively, and so slice ${ }^{+}$implies mod-slice. The converse implication is true under weaker assumptions, as the following lemma indicates.

Lemma 4.3. Suppose $(X, d, \mu)$ is a minimally nice, upper $Q$-regular, metric measure space, and that $(x, y ; \gamma) \in \bmod -\operatorname{slice}(C, c)$ for some $x, y \in X$, $\gamma \in \Gamma(x, y)$. Then there exists $C^{\prime}$ dependent only on $C, c, Q$, and $C^{+}(\mu, Q)$ such that $(x, y ; \gamma) \in \operatorname{slice}^{+}\left(C^{\prime}, c\right)$.

Proof. We need to prove $\left(\mathrm{Sli}_{1}^{+}\right)$. If $\lambda_{i}$ intersects $5 E_{i}$, it suffices to appeal to $\left(\mathrm{Sli}_{4}^{+}\right)$. If, on the other hand, $\lambda_{i}$ is disjoint from $5 E_{i}$, we let $E=E_{i}, F=\lambda_{i}$, and apply Lemma 7.3 to $\left(\mathrm{Sli}_{5}\right)$.

The following lemma formulates the interdependence of the constants $C$ and $c$ in the mod-slice $(C, c)$ condition as the constant $c$ is allowed to shrink.

Lemma 4.4. Suppose $(X, d, \mu)$ is a minimally nice, locally $\left(Q, \kappa, \delta_{0}, \psi\right)$ Loewner, metric measure space. Let $0<c^{\prime} \leq c \leq \delta_{0} / 5$. If $(x, y ; \gamma) \in$ mod-slice $(C, c)$ for some $x, y \in X, \gamma \in \Gamma(x, y)$, then $(x, y ; \gamma) \in$ mod-slice $\left(C^{\prime}, c^{\prime}\right)$, where

$$
C^{\prime}=3^{Q}\left[C \vee \psi(6)^{-1} \vee \psi\left(3 c / c^{\prime}\right)^{-1}\right] .
$$

Proof. Except for (Sli5), all the defining conditions for $\left(C^{\prime}, c^{\prime}\right)$-mod-slice follow from those for $(C, c)$-mod-slice. For $\left(\mathrm{Sli}_{5}\right)$, we choose the same data $\left\{S_{i}, d_{i}, x_{i}\right\}_{i=0}^{m}$ for both choices of constants. Fixing $\lambda \in \Gamma(x, y)$, we also choose the same subpaths $\lambda_{i}$ unless otherwise stated. Fix $0<i<m$, and write $E_{i}=\bar{B}_{d}\left(x_{i}, c d\left(x_{i}\right)\right), E_{i}^{\prime}=\bar{B}_{d}\left(x_{i}, c^{\prime} d\left(x_{i}\right)\right)$. Suppose first that $\lambda_{i}$ intersects $3 E_{i}$. In view of $\left(\mathrm{Sli}_{4}^{+}\right)$, we may select a segment $\lambda_{i}^{\prime}$ of $\lambda_{i}$ that connects $3 E_{i}$ with $\partial\left(4 E_{i}\right)$ and lies inside $5 E_{i}$. Since $\operatorname{dia}_{d}\left(\lambda_{i}^{\prime}\right) \geq c d\left(x_{i}\right)$, the local Loewner property with $E=E_{i}^{\prime}$ and $F=\left(\lambda_{i}^{\prime}\right)$ implies that

$$
\psi\left(3 c / c^{\prime}\right) \leq \bmod _{Q}(E, F ; B(\epsilon \kappa d(x))) \leq \bmod _{Q}(E, F),
$$

as required (note that $\psi$ is nonincreasing by definition).

Suppose instead that $\lambda$ is a path for which $\lambda_{i} \cap 3 E_{i}=\emptyset$. We first prove this case under the added assumption $c^{\prime} \leq c / 6$. Then the data

$$
\left(E, E^{\prime}, F, x, r\right)=\left(\overline{E_{i} \backslash(5 / 6) E_{i}}, E_{i}^{\prime}, \lambda_{i}, x_{i}, c d\left(x_{i}\right)\right)
$$

satisfy the assumptions of Lemma 7.4. This is clear except for the inequality $\operatorname{dia}_{d}(E) \leq 3 \operatorname{dist}_{d}\left(E, E^{\prime}\right)$, which follows from the estimates $\operatorname{dia}_{d}(E) \leq 2 r$ and $\operatorname{dist}_{d}\left(E, E^{\prime}\right) \geq 2 r / 3$.

Now, $\operatorname{dia}_{d}(E) \geq r / 6$. To see this, we simply choose an initial path $\eta$ from $x_{i}$ to a point $x^{\prime}$ with $d\left(x^{\prime}\right)<d\left(x_{i}\right) / 2$, and extract a subpath $v=\eta[u, v]$ that 
lies in $E$, with $d\left(u, x_{i}\right)=5 r / 6$ and $d\left(v, x_{i}\right)=r$. Then $\operatorname{dia}_{d}(E) \geq \operatorname{dia}_{d}(v) \geq$ $r / 6$.

It follows similarly that $\operatorname{dia}\left(E^{\prime}\right) \geq c^{\prime} d(x)$, and it is clear that $\operatorname{dist}_{d}\left(E, E^{\prime}\right)$ $<r$. Thus

$$
\Delta_{d}\left(E, E^{\prime}\right) \leq \frac{c d\left(x_{i}\right)}{c^{\prime} d\left(x_{i}\right)}=\frac{c}{c^{\prime}} .
$$

Using Lemma 7.4 and the estimate $\bmod _{Q}\left(\lambda_{i}, E_{i}\right) \geq C^{-1}$, we deduce that $\bmod _{Q}\left(E^{\prime}, F\right) \geq 3^{-Q}\left[\psi(3) \wedge C^{-1} \wedge \psi\left(c / c^{\prime}\right)\right]$. This gives the desired conclusion for such paths $\lambda$ with a constant

$$
C^{\prime}=3^{Q}\left[C \vee \psi(3)^{-1} \vee \psi\left(c / c^{\prime}\right)^{-1}\right],
$$

which is stronger than the desired conclusion.

It remains to prove it for this second type of path when $c^{\prime}>c / 6$. Since the modulus in $\left(\mathrm{Sli}_{5}\right)$ increases as $c^{\prime}$ increases, any $C^{\prime}$ that works for $c^{\prime}=c / 6$ also works for $c^{\prime}>c / 6$. But for $c^{\prime}=c / 6$, and the type of path that we need to consider, we can take

$$
C^{\prime}=3^{Q}\left[C \vee \psi(3)^{-1} \vee \psi(6)^{-1}\right]=3^{Q}\left[C \vee \psi(6)^{-1}\right] .
$$

The desired estimate thus follows for larger $c^{\prime}$.

Suppose $C \geq 1$. Given points $x, y \in X$, and a path $\gamma \in \Gamma(x, y), \gamma$ : $[0, L] \rightarrow X$, we say that $\gamma$ is a $C$-uniform path for $x, y$ if $\operatorname{len}_{d}(\gamma) \leq C d(x, y)$ and

$$
\forall t \in[0, L]: \quad d_{X}(\gamma(t)) \geq C^{-1}\left[\operatorname{len}_{d}\left(\left.\gamma\right|_{[0, t]}\right) \wedge \operatorname{len}_{d}\left(\left.\gamma\right|_{[t, L]}\right)\right] .
$$

If we assume, as we may, that $\gamma$ is parametrized by arclength, the second condition above takes the simpler form

$$
\forall t \in(0, L): \quad d_{X}(\gamma(t)) \geq C^{-1}[t \wedge(L-t)] .
$$

We say that $X$ is a $C$-uniform space if there is a $C$-uniform path for every pair of points $x, y \in X$. It is also useful to extend the concept to paths $\gamma:[0, L] \rightarrow \bar{X}$ parametrized by arclength. We say that such a path is a $C$ uniform path if $L \leq C d_{\bar{X}}(\gamma(0), \gamma(L))$ and (4.5) holds. Equivalently, modulo a controlled change of constants, $\gamma$ is a uniform path if for some constant $C^{\prime}$, the path segments $\left.\gamma\right|_{[\epsilon, L-\epsilon]}$ are $C^{\prime}$-uniform for all $0<\epsilon<L / C^{\prime}$.

We now show that uniform spaces satisfy a slice ${ }^{+}$condition; a similar but simpler proof for a somewhat different slice condition in the Euclidean setting can be found in [BuKo2, Theorem 3.2].

Theorem 4.6. Suppose $(X, d)$ is a $C$-uniform space. Then it also satisfies a $\left(C^{\prime}, c^{\prime}\right)$-slice ${ }^{+}$condition, for some $C^{\prime}, c^{\prime}$ dependent only on $C$. Additionally, if $x, y \in X$ and $\gamma \in \Gamma(x, y)$ is a geodesic segment, then $(x, y ; \gamma) \in \operatorname{slice}^{+}\left(C^{\prime \prime}, c^{\prime \prime}\right)$, for some $C^{\prime \prime}, c^{\prime \prime}$ dependent only on $C$. 
Proof. Let us fix $x, y \in X$. By symmetry, we may assume that $d(y) \leq d(x)$. Let $\gamma=[x, y]$ be a geodesic path. By [BoHeKo, Theorem 2.10], $\gamma$ is a $C_{1}$-uniform path for the pair $x, y$, with $C_{1}=C_{1}(C) \geq 1$. We assume, as we may, that $\gamma:[0, l] \rightarrow X$ is parametrized by arclength and that $C_{1} \geq C$. Since we shall only use the fact that $\gamma$ is a $C_{1}$-uniform path, the first conclusion in the theorem thus follows from the second which we now prove.

Let $\alpha=5 / 4$ and let $n_{0}$ be the least integer such that $\alpha^{n_{0}}>3 C_{1}$; in particular, $n_{0}>\log _{\alpha} 3>4$. For $z \in\{x, y\}$, define $d$-balls $B_{i}^{z}=B\left(z, r_{i}^{z}\right)$ for each non-negative integer $i$, where each $r_{i}^{z}$ is a number chosen so that

$$
\frac{9}{10} \cdot \frac{\alpha^{i} d(z)}{25 C} \leq r_{i}^{z} \leq \frac{\alpha^{i} d(z)}{25 C}
$$

and

$$
\operatorname{len}_{d}\left(\gamma \cap \partial B\left(z, r_{i}^{z}\right)\right)=0 .
$$

Such numbers $r_{i}^{z}$ exist; indeed, the finiteness of $\operatorname{len}_{d}(\gamma)$ implies that the length of the part of $\gamma$ lying on the sphere $\partial B_{d}(z, r)=\{w \in X \mid d(w, z)=r\}$, is zero for almost every $r>0$. Note that

$$
\frac{9 \alpha^{j}}{10} \leq \frac{r_{i+j}^{z}}{r_{i}^{z}} \leq \frac{10 \alpha^{j}}{9}
$$

We also define the associated annuli

$$
A_{i}^{z}= \begin{cases}B_{0}^{z} & i=0, \\ B_{i}^{z} \backslash \overline{B_{i-1}^{z}}, & i \in \mathbb{N} .\end{cases}
$$

We may assume that $d(x, y) \geq d(x) / 2 C$, since otherwise the single slice $S_{0}=B\left(x, r_{N}^{x}\right)$ suffices, where $N$ is any integer such that $\alpha^{N}>20$. For each $i \geq 1$, there is a non-negative integer $g(i)$ such that $B_{i}^{x}$ intersects $B_{j}^{y}$ precisely when $j \geq g(i)$. A little calculation shows that $r_{g(i)}^{y} / r_{i}^{x} \geq \alpha$ for $i \leq 2$, while $r_{g(i)}^{y} / r_{i}^{x}<1$ for all sufficiently large $i$. Let $i_{0} \geq 3$ to be the least integer $i$ for which $r_{g(i)}^{y} / r_{i}^{x}<5$. Note that $r_{g\left(i_{0}-1\right)-1}<d(x, y)$, and so $r_{g\left(i_{0}-1\right)}^{y}<25 d(x, y) / 18$ and $r_{i_{0}-1}^{x}<5 d(x, y) / 18$. Using (4.7) again, we see that $r_{i_{0}}^{x}<d(x, y) / 2$. Since we always have $r_{i}^{x}+r_{g(i)}^{y}>d(x, y)$, it follows that $r_{g\left(i_{0}\right)}^{y}>d(x, y) / 2$, and so $r_{g\left(i_{0}\right)}^{y} / r_{i_{0}}^{x} \in(1,5)$. Clearly also $g\left(i_{0}\right) \geq 3$. Let $m=i_{0}+g\left(i_{0}\right)$ and define the slices

$$
S_{i}= \begin{cases}A_{i}^{x}, & 0 \leq i \leq i_{0}, \\ A_{m-i}^{y}, & i_{0}+2 \leq i \leq m \\ B_{g\left(i_{0}\right)+n_{0}}^{y} \backslash\left(S_{i_{0}} \cup S_{i_{0}+2}\right), & i=i_{0}+1 .\end{cases}
$$

We choose $x_{0}=x, x_{m}=y$, and pick $x_{i} \in \gamma$ so that $d\left(x_{i}, x\right)=\left(r_{i}^{x}+r_{i-1}^{x}\right) / 2$ for $0<i \leq i_{0}$, and $d\left(x_{m-i}, y\right)=\left(r_{i}^{y}+r_{i-1}^{y}\right) / 2$ for $0<i \leq g\left(i_{0}\right)-1$. 
Condition $\left(\mathrm{Sli}_{4}^{+}\right)$is easy to deduce in all cases. As for $\left(\mathrm{Sli}_{3}\right)$, we first combine (4.7) with the definition of $n_{0}$ and the fact that $r_{g\left(i_{0}\right)}>d(x, y) / 2$, to deduce that $B_{g\left(i_{0}\right)+n_{0}}^{y}$ has radius larger than $C_{1} d(x, y)$. It therefore fully contains $\gamma$, and $\left(\mathrm{Sli}_{3}\right)$ is now easy to verify.

Let us consider $\left(\mathrm{Sli}_{2}\right)$ for $1 \leq i \leq i_{0}$. Define $t_{i}^{-}$and $t_{i}^{+}$to be the values of $t$ for which $\gamma(t)$ is the point of first entry into, and last exit from, $\overline{S_{i}}$, respectively. Uniformity ensures that $\gamma(t)$ cannot lie in $B_{d}\left(x, r_{i-1}^{x}\right)$ for any $t \geq C_{1}\left(d(x)+r_{i-1}^{x}\right)$, so $t_{i}^{-} \leq C_{1}\left(d(x)+r_{i-1}^{x}\right) \lesssim r_{i}^{x}$. Similarly, $t_{i}^{+} \lesssim r_{i}^{+}$, and so $t_{i}^{+}-t_{i}^{-} \lesssim r_{i}^{x}$. But trivially $t_{i}^{-} \geq r_{i-1}^{x}$ and, since $r_{i_{0}}^{x}<d(x, y) / 2$, we also have $l-t_{i}^{+}>d(x, y) / 2 \gtrsim r_{i}^{x}$. Uniformity therefore ensures that $d(\gamma(t)) \gtrsim r_{i}^{x}$ for all $t \in\left[t_{i}^{-}, t_{i}^{+}\right]$. This lower bound together with the upper bound on $t_{i}^{+}-t_{i}^{-}$imply $\left(\mathrm{Sli}_{2}\right)$.

Condition $\left(\mathrm{Sli}_{1}^{+}\right)$follows readily from the construction. The "middle slice" $S_{i_{0}+1}$ deserves separate attention. Here it is important that the succeeding slices are annuli $A_{i}^{y}$, where $i \leq g\left(i_{0}\right)-2$; the gap between this upper index and $g\left(i_{0}\right)$ ensures $\left(\mathrm{Sli}_{1}^{+}\right)$in this case. In each case, it is in fact clear that every path $\lambda \in \Gamma(x, y)$ contains a closed subpath $\lambda_{i}$ whose diameter is comparable with the diameter of the slice; for instance if $i \neq i_{0}+1,0, m$ and so $S_{i}$ is an annulus, $\lambda_{i}$ is any subpath that connects the inner spherical boundary of $S_{i}$ to its outer spherical boundary.

We now pause to recall some recent results of Bonk, Heinonen, and Koskela [BoHeKo] that we shall need. If $(X, d)$ is a minimally nice metric space, and $w \in X$ is a base point, we define the densities $\rho_{\epsilon}(x)=$ $\exp (-\epsilon k(x, w))$ and $\sigma_{\epsilon}(x)=\rho_{\epsilon}(x) / d(x)$, for all $\epsilon>0$. This gives rise to the metric space ${ }^{1} X_{\epsilon}=\left(X, d_{\epsilon}\right)$ with

$$
d_{\epsilon}(x, y)=\inf _{\gamma \in \Gamma(x, y)} \int_{\gamma} \rho_{\epsilon}(z)\left|d_{k} z\right|=\inf _{\gamma \in \Gamma(x, y)} \int_{\gamma} \sigma_{\epsilon}(z)|d z|
$$

where $\left|d_{k} z\right|=|d z| / d_{X}(z)$ denotes the quasihyperbolic distance element. According to $[\mathrm{BoHeKo}]$, the resulting space is incomplete and so we can associate a boundary distance $d_{\epsilon}(\cdot)$ and a quasihyperbolic metric $k_{\epsilon}$ with $d_{\epsilon}$, as we did with $d$.

If $\mu$ is a Borel measure on $X$, and $Q \geq 1$ is fixed, we also attach the Borel measure $d \mu_{\epsilon}(z)=\sigma_{\epsilon}(z)^{Q} d \mu(z)$ to $\left(X, \bar{d}_{\epsilon}\right)$; the parameter $Q$ is omitted from this notation, since it will always be given by a (local or global) $Q$-Loewner condition. The point of this definition is that the $Q$-modulus of a path family is invariant under the identity map $i_{\epsilon}: X \rightarrow X_{\epsilon}$, as is immediate from the definition. We write $X_{\epsilon}$ as a shortcut for either $\left(X, d_{\epsilon}\right)$ and $\left(X, d_{\epsilon}, \mu_{\epsilon}\right)$.

We now state Theorem 6.39 of [BoHeKo] as a lemma. In this lemma, the metric measure space $\left(X, d_{\rho}, \mu_{\rho}\right)$ is derived from the metric measure space $(X, d, \mu)$ via some density $\rho$. We have already defined the metric $d_{\rho}$ in the

${ }^{1}$ For consistency with Sect. 0, we would need to write $d_{\sigma_{\epsilon}}$ instead of $d_{\epsilon}$. For ease of notation, however, we insist that $d_{\epsilon}$ is henceforth defined as in (4.8). 
introduction, and $d \mu_{\rho}$ is simply $\rho^{Q} d \mu$. Also the notation $d_{\rho}(x)$ stands for the $d_{\rho}$-distance to the boundary of a point $x \in X$.

Lemma 4.9. Let $(X, d, \mu)$ be a locally compact, incomplete, quasiconvex locally $Q$-Loewner space. Assume that $\rho: X \rightarrow(0, \infty)$ is a continuous function such that the following two conditions hold for some $C \geq 1$ :

$$
\frac{1}{C} \leq \frac{\rho(x)}{\rho(y)} \leq C, \quad x, y \in B_{d}(z, d(z) / 2), z \in X,
$$

and

$$
\frac{1}{C} \rho(x) d(x) \leq d_{\rho}(x) \leq C \rho(x) d(x), \quad x \in X .
$$

Then $\left(X, d_{\rho}, \mu_{\rho}\right)$ is a locally $Q$-Loewner space, quantitatively.

We actually need a stronger "more local" version of this lemma. Specifically, we want a version with the same conclusion but with only a local version of the quasiconvexity assumption, and with the ball in (4.10) replaced by a smaller ball $B_{d}(z, c d(z))$ for some fixed but arbitrary $c>0$. It is a routine matter to modify the proof in $[\mathrm{BoHeKo}]$ to prove this stronger version of the lemma.

Our next lemma is a combination of Propositions 4.5 and 4.37 of [BoHeKo].

Lemma 4.12. Suppose $(X, d)$ is minimally nice, and that $(X, k)$ is both $C$ Gromov hyperbolic and $K$-roughly starlike, for some $C \geq 1, K>0$. Then $X_{\epsilon}$ has diameter at most $2 / \epsilon$ and there are positive numbers $C^{\prime}, \epsilon$ dependent only on $C, K$ such that $X_{\epsilon}$ is $C^{\prime}$-uniform for all $0<\epsilon \leq \epsilon_{0}$. Furthermore, there exists $c=c(C, K) \in(0,1)$ such that the quasihyperbolic metrics $k$ and $k_{\epsilon}$ satisfy the quasi-isometric condition

$$
c \epsilon k(x, y) \leq k_{\epsilon}(x, y) \leq e \epsilon k(x, y) .
$$

Using Theorem 4.6 and Lemma 4.12, we know that $X_{\epsilon}$ satisfies a slice $^{+}$ condition if $\epsilon$ is sufficiently small. We shall transport this condition over to $X$ by means of a modulus argument. For such an argument to work, we need to show that $X_{\epsilon}$ is a Loewner space.

Lemma 4.13. Suppose that $(X, d, \mu)$ is minimally nice, locally $Q$-regular, and locally $Q$-Loewner, and that $(X, k)$ is Gromov hyperbolic and roughly starlike. Then $X_{\epsilon}$ is $Q$-Loewner for all $0<\epsilon \leq \epsilon_{0}$, where $\epsilon_{0}$ depends quantitatively on the hypotheses, and the Loewner data depend quantitatively on $\epsilon$ and the hypotheses.

Proof. According to Theorem 6.4 of [BoHeKo], a uniform and local $Q$ Loewner space is $Q$-Loewner, quantitatively. But by Lemma 4.12, $X_{\epsilon}$ is uniform when $\epsilon$ is sufficiently small. Thus it suffices to show that $X_{\epsilon}$ is locally $Q$-Loewner when $\epsilon$ is small enough. The local Loewner condition 
will in turn follow by applying the stronger version of Lemma 4.9 (defined after the statement of that lemma) to the density $\rho=\sigma_{\epsilon}$. We must therefore show that this density satisfies the hypotheses of that lemma.

A regular Loewner space is quasiconvex, as proven in [HeKo, Theorem 3.13]. This proof is readily modified to show that a locally regular, locally Loewner space is locally $C_{1}$-quasiconvex, where $C_{1} \geq 1$ is dependent only on the local regularity and Loewner data.

Let us fix an arbitrary point $x \in X$, and write $B=B_{d}\left(x, d(x) / 4 C_{1}\right)$. We next show that

$$
\frac{k(y, z)}{2 C_{1}} \leq \frac{d(y, z)}{d(x)}, \quad y, z \in B .
$$

Suppose $y, z \in B$. Since $d(y, z) \leq d(x) / 2 C_{1}$, it follows by quasiconvexity that there is a path $\lambda \in \Gamma(y, z)$ such that $\operatorname{len}_{d}(\lambda) \leq C_{1} d(y, z)$. Since $\lambda$ is of length at most $d(x) / 2$, the set $\lambda$ must be contained in $B^{\prime} \equiv B(x, d(x) / 2)$. But $d(z) \geq d(x) / 2$ on $B^{\prime}$, so len $k(\lambda) \leq 2 \operatorname{len}_{d}(\lambda) / d(x)$, which implies (4.14).

Assuming that $\epsilon \leq \epsilon_{0} \leq 1 / e$ is small enough that Lemma 4.12 is valid, and taking $y=x$ in (4.14), we see that $B \subset B_{k}(x, 1 / 2) \subset B_{k_{\epsilon}}(x, 1 / 2)$. By (2.8), we see that $d(z) / d(x)$ lies in the interval $\left(e^{-1 / 2}, e^{1 / 2}\right)$ when $z \in B$. Using the triangle inequality we see that the density function $\rho_{\epsilon}$ satisfies the estimate

$$
\exp (-\epsilon k(u, v)) \leq \frac{\rho_{\epsilon}(u)}{\rho_{\epsilon}(v)} \leq \exp (\epsilon k(u, v)), \quad u, v \in X .
$$

Since $\epsilon \leq 1 / e$, it follows that $\rho_{\epsilon}(z) / \rho_{\epsilon}(x) \in\left[e^{-1 /(2 e)}, e^{1 /(2 e)}\right]$ for all $z \in B$. We deduce that

$$
\frac{1}{e^{2}} \leq \frac{\sigma_{\epsilon}(y)}{\sigma_{\epsilon}(z)} \leq e^{2}, \quad y, z \in B .
$$

Since $(X, k)$ is $K$-roughly starlike, Gromov hyperbolic, and proper, it follows from Lemma 4.16 of [BoHeKo] that there are constants $0<c_{2}$ $<C_{2}$, dependent only on $\epsilon$ and $K$, such that

$$
c_{2} \rho_{\epsilon}(x) \leq d_{\epsilon}(x) \leq C_{2} \rho_{\epsilon}(x), \quad x \in X,
$$

which can be rewritten as

$$
c_{2} \sigma_{\epsilon}(x) d(x) \leq d_{\epsilon}(x) \leq C_{2} \sigma_{\epsilon}(x) d(x), \quad x \in X .
$$

We have now verified all of the hypotheses of the stronger version of Lemma 4.9, and so the proof can be completed as indicated above.

By Theorem 4.6 and the uniformity of $X_{\epsilon}$ for small $\epsilon>0$, we already know that $X_{\epsilon}$ satisfies a slice ${ }^{+}$condition for appropriately chosen $\epsilon>0$. The proof of Theorem 4.2, which we now present, consists of showing that the slice ${ }^{+}$condition on $X_{\epsilon}$ induces a slice condition on $X$. In this proof, $\bmod _{Q}$ and $\bmod _{\epsilon, Q}$ denote conformal modulus with respect to $d$ and $d_{\epsilon}$, respectively. 
Note. There are quite a few constants in this proof. It is convenient to denote each of them as $C_{*}$ or $c_{*}$, possibly with some prime superscripts, where $*$ is some suggestive symbol (rather than a variable, as is the case elsewhere in this paper). For example, $C_{\epsilon}$ and $C_{1}$ are completely unrelated constants, irrespective of whether or not the related variable $\epsilon$ takes on the value 1 .

Proof of Theorem 4.2. We wish to give slice ${ }^{+}$data for a fixed but arbitrary pair of points $x, y \in X$ and $k$-geodesic $[x, y]$. Applying Lemmas 4.12 and 4.13, we choose $\epsilon_{0}>0$ so small that $X_{\epsilon}$ is both uniform and $Q$-Loewner for all $0<\epsilon \leq \epsilon_{0}$. Lemma 4.12 also implies that $[x, y]$ is a $\left(k_{\epsilon} ; C_{\mathrm{QG}}\right)$ quasigeodesic; $C_{\mathrm{QG}}$ depends only on the Gromov hyperbolicity and rough starlikeness constants, $C_{\mathrm{G}}$ and $C_{\mathrm{S}}$, respectively.

Fixing $\epsilon=\epsilon_{0}$, Theorem 4.6 allows us to choose data $\left\{S_{i ; \epsilon}, x_{i ; \epsilon}\right\}_{i=0}^{m}$ associated with the slice ${ }^{+}\left(C_{\epsilon}, c_{\epsilon}\right)$ condition for $x, y \in X_{\epsilon}$ and the $k_{\epsilon^{-}}$ quasigeodesic $[x, y]$. By the Loewner assumption, the mod-slice $\left(C_{\epsilon}^{\prime}, c_{\epsilon}\right)$ condition also follows for some $C_{\epsilon}^{\prime} \geq 6$, and $c_{\epsilon}=\delta_{0} / 4$, where $\delta_{0}$ is the third local Loewner parameter. We then define the $C_{d}$-slice ${ }^{+}$data for $x, y \in X$ and the $k$-geodesic $[x, y]$ to be $\left\{S_{i}, x_{i}\right\}_{i=0}^{m}$, where $x_{i}=x_{i ; \epsilon}$, $S_{i}=S_{i ; \epsilon} \cap B_{d}\left(x_{i}, C_{d} d\left(x_{i}\right)\right) ; C_{d} \geq 6$ and $0<c_{d}<1$ will be specified later. Consistent with the slice ${ }^{+}$definition, we also write $E_{i ; \epsilon}=\bar{B}_{d_{\epsilon}}\left(x_{i}, c_{\epsilon} d_{\epsilon}\left(x_{i}\right)\right)$ and $E_{i}=\bar{B}_{d}\left(x_{i}, c_{d} d\left(x_{i}\right)\right)$.

By Lemma 4.12, there exists a constant $c_{1} \geq 1$ such that $\operatorname{dia}_{k}([x, y] \cap$ $\left.S_{i ; \epsilon}\right) \leq c_{1}$. Appealing to (2.7), we see that there exists a constant $C_{1}$ such that $[x, y]$ is contained in $\bigcup_{i=0}^{m} B_{d}\left(x_{i}, C_{1} d\left(x_{i}\right)\right)$, where $C_{1}=\exp \left(c_{1}\right)-1 \geq c_{1}$. Conditions $\left(\mathrm{Sli}_{2}\right)$ and $\left(\mathrm{Sli}_{3}\right)$ are now clearly true for any choice of constant $C_{d} \geq C_{1}$.

Using (2.7), Lemma 4.12, and (2.9) in that order, we see that there are constants $c_{2, \epsilon}, c_{2}, c_{d} \in(0,1)$, dependent only on $\epsilon, C_{\epsilon}, C_{\mathrm{G}}, C_{\mathrm{S}}$, such that

$$
6 E_{i ; \epsilon} \supset B_{k_{\epsilon}}\left(x_{i}, c_{2, \epsilon}\right) \supset B_{k}\left(x_{i}, c_{2}\right) \supset \bar{B}_{d}\left(x_{i}, 6 c_{d} d\left(x_{i}\right)\right) \supset 6 E_{i} .
$$

This immediately implies $\left(\mathrm{Sli}_{4}\right)$ for any choice of constant $C_{d} \geq c_{2}^{-1}$ (as well as $\left(\mathrm{Sli}_{4}^{+}\right)$for any $c_{d} \leq c_{2}$, but this does not concern us).

It remains only to prove $\left(\mathrm{Sli}_{1}^{+}\right)$for some choice of $C_{d}$. In a similar fashion to (4.17), we can show that there exists a constant $c_{3} \in(0,1)$, dependent on the same parameters as $c_{d}$, such that $c_{3} E_{i ; \epsilon} \subset E_{i}$. Assume therefore that a path $\lambda \in \Gamma(x, y)$ is given, and let $\lambda_{i ; \epsilon}$ be the subpath provided by ( $\left.\mathrm{Sli}_{5}\right)$. By Lemma 4.4, there exists some $c^{\prime} \in(0,1)$ such that $\bmod _{\epsilon, Q}\left(\lambda_{i, \epsilon}, E_{i}\right) \geq$ $\bmod _{\epsilon, Q}\left(\lambda_{i ; \epsilon}, c_{3} E_{i ; \epsilon}\right) \geq c^{\prime}$. Using Lemma 7.3, we see that there exists some $C_{2}$ dependent only on allowed parameters such that $\lambda_{i ; \epsilon}$ must intersect $B\left(x, C_{2} d\left(x_{i}\right)\right)$. We now finally declare $C_{d}=C_{1} \vee c_{2}^{-1} \vee 2 C_{2}$.

If $\lambda_{i ; \epsilon}$ lies fully in $B_{d}\left(x_{i}, C_{d} d\left(x_{i}\right)\right)$, then take $\lambda_{i}=\lambda_{i ; \epsilon}$. By Lemma 4.4, we have $\bmod _{\epsilon, Q}\left(\lambda_{i}, E_{i}\right) \geq \bmod _{\epsilon, Q}\left(\lambda_{i}, C_{d}^{-1} E_{i: \epsilon}\right) \geq c^{\prime}$, for some $c^{\prime}>0$ dependent only the data in the hypotheses. The identity map from $(X, d)$ to 
$\left(X, d_{\epsilon}\right)$ is locally a dilation composed with a controlled bilipschitz distortion and so it clearly satisfies (7.6). By Lemma 7.5, it follows that $\bmod _{Q}\left(\lambda_{i}, E_{i}\right) \geq c^{\prime \prime}$ for some $c^{\prime \prime}>0$ dependent only the data in the hypotheses. Condition $\left(\mathrm{Sli}_{1}^{+}\right)$now follows as in Lemma 4.3.

Alternatively if $\lambda_{i ; \epsilon}$ does not lie fully in $B_{d}\left(x_{i}, C_{d} d\left(x_{i}\right)\right)$, then we choose a subpath $\lambda_{i}$ of $\lambda_{i ; \epsilon}$ which connects points $v$, w, where $d\left(v, x_{i}\right)=C_{2} d\left(x_{i}\right)$ and $d\left(w, x_{i}\right)=2 C_{2} d\left(x_{i}\right)$, and so $\left(\mathrm{Sli}_{1}^{+}\right)$is clearly true.

We now give a more explicit description of a class of spaces for which kG-hyperbolicity implies a slice condition.

Theorem 4.18. Suppose $\left(Y, d_{Y}, \mu_{Y}\right)$ is a complete, $Q$-regular, $Q$-Loewner metric measure space, and that $X \subsetneq Y$ is a bounded open connected subset of $Y$. Let d denote the inner metric (on $X$ ) associated with $\left.d_{X} \equiv d_{Y}\right|_{X \times X}$ and let $\mu$ be the restriction of $\mu_{Y}$ to $X$. If $(X, d)$ is $k G$-hyperbolic, then it satisfies a slice ${ }^{+}$condition, quantitatively.

As we already indicated at the beginning of the previous section, there are many examples of metric measure spaces $\left(Y, d_{Y}, \mu_{Y}\right)$ that satisfy the assumptions of this theorem. Examples include $n$-regular Riemannian manifolds of non-negative Ricci curvature, Carnot-Carathéodory spaces such as the Heisenberg group; see [HeKo, Sect. 6]. Also noteworthy are the exotic examples with non-integer dimension $Q$ given by Bourdon and Pajot [BoPa] and by Laakso [Lk].

It is easily verified that, under the hypotheses of Theorem 4.18, upper regularity, local regularity, and the local Loewner property are inherited by $\left(X, d_{X}, \mu\right)$ and hence by $(X, d, \mu)$. Additionally, $Y$ (and hence $\left.X\right)$ is locally compact because it is complete and $Q$-regular. Thus, in view of Theorem 4.1, the proof of Theorem 4.18 reduces to proving the following result.

Proposition 4.19. Let $(X, d, \mu)$ be as in Theorem 4.18 and let $M=$ $\sup _{z \in X} d(z)$. Then there exists a number $K$, dependent quantitatively on the hypotheses, such that $(X, k)$ is $K$-roughly starlike with respect to any basepoint $w$ for which $d(w)>M / 2$.

Proof. By Theorem 3.1, it suffices to verify a $Q L(C)$ condition. Condition $Q L(C)$ (a) follows readily from the second conclusion in Lemma 7.1 applied to the ambient space $Y$; note that since $\gamma$ does not intercept $\partial X$, it lies in $X$. Condition $Q L(C)$ (b) is almost as easy: we make some tentative choice of $y \in A(z, r, 4 C) \cap \partial X$ and again apply the second conclusion in Lemma 7.1. Since we do not want $\gamma$ to wander outside $\bar{X}$, we cut it off when it first reaches a point on $\partial X$, and redefine $y$ to be that point. For $Q L(C)(\mathrm{c})$, we apply the first conclusion in Lemma 7.1 with $y \in \partial X, d(x, y)<2 d(x)$, and cut off a near minimal-length connecting path when it first reaches a point $x^{\prime}$ with $d\left(x^{\prime}\right)=d(x) / 2$. For all parts, we can take $C=2 C_{0}$. Thus Proposition 4.19 follows from Theorem 3.1. 


\section{Basic properties of GHS spaces}

We begin by introducing some extra notation that we shall use in these two sections. We say that $(X, d)$ is a $C$-GHS space, $C \geq 1$, if it is an incomplete locally compact length space (and so minimally nice) and it satisfies both a $C$-Gehring-Hayman and a $C$-separation condition. We denote by $\lambda_{x, y}$ any path from $x$ to $y$ such that $\operatorname{len}_{d}\left(\lambda_{x, y}\right)$ is "close to" $d(x, y)$.

Lemma 5.1. Suppose that $(X, d)$ satisfies a $C$-Gehring-Hayman condition. Then whenever $x, y \in X$, and $w$ lies on a geodesic $[x, y]$, we have

$$
d(x, y) \geq 2 d(x, w) /(C+1) .
$$

Proof. If the conclusion were false, then

$$
\operatorname{len}_{d}([x, w]) \geq d(x, w)>\frac{C+1}{2} d(x, y)
$$

and

$$
\operatorname{len}_{d}([w, y]) \geq d(w, y) \geq d(x, w)-d(x, y)>\frac{C-1}{2} d(x, y) .
$$

Combining the two inequalities we have

$$
\begin{aligned}
\operatorname{len}_{d}([x, y]) & =\operatorname{len}_{d}([x, w])+\operatorname{len}_{d}([w, y]) \\
& >\left(\frac{C+1}{2}+\frac{C-1}{2}\right) d(x, y)=C d(x, y),
\end{aligned}
$$

contradicting $(\mathrm{GH})$.

Lemma 5.3. Suppose that $X$ is a $C$-GHS space and that $x, y, z \in X$. Suppose further that $[a, b] \subset[x, z],\left[a^{\prime}, b^{\prime}\right] \subset[z, y]$. Then

$$
\begin{aligned}
\operatorname{len}_{d}([a, b]) & \leq 2 C d_{1}+C^{2}(C+1) d_{2} / 2, \\
\operatorname{len}_{d}\left(\left[a^{\prime}, b^{\prime}\right]\right) & \leq C(C+5) d_{1} / 2+C^{2}(C+1) d_{2} / 2,
\end{aligned}
$$

where $d\left(a, a^{\prime}\right) \vee d\left(b, b^{\prime}\right)=d_{1}$ and $d\left(a^{\prime}\right)=d_{2}$.

Note. As mentioned in Sect. 1 , the inclusions $[a, b] \subset[x, z]$ and $\left[a^{\prime}, b^{\prime}\right] \subset$ $[z, y]$ are meant with the ordering convention coming from the parametrization of the geodesics by the real interval, so that $b \in[a, z]$ and $b^{\prime} \in\left[a^{\prime}, y\right]$.

Proof of Lemma 5.3. We choose a path $\lambda_{b^{\prime}, b} \in \Gamma\left(b^{\prime}, b\right)$ such that len ${ }_{d}\left(\lambda_{b^{\prime}, b}\right)$ $\leq d_{1}+\epsilon$, where $\epsilon>0$ is fixed but arbitrary. By $\left(\operatorname{Sep}_{2}\right)$, there exists a point $a^{\prime \prime} \in S_{a^{\prime}}^{[y, z]} \cap \lambda$, where $\lambda=\left[y, b^{\prime}\right]+\lambda_{b^{\prime}, b}+[b, z] \in \Gamma(y, z)$. By $\left(\operatorname{Sep}_{1}\right)$, we have $d\left(a^{\prime}, a^{\prime \prime}\right)<C d_{2}$. If $a^{\prime \prime} \in\left[y, b^{\prime}\right]$ then by (GH)

$$
\operatorname{len}_{d}\left(\left[a^{\prime}, b^{\prime}\right]\right) \leq \operatorname{len}_{d}\left(\left[a^{\prime}, a^{\prime \prime}\right]\right)<C^{2} d_{2},
$$


which proves the second inequality in our claim. Using (GH) and (5.2), it follows that

$$
\begin{aligned}
\operatorname{len}_{d}([a, b]) \leq C d(a, b) & \leq C\left(d\left(a, a^{\prime}\right)+d\left(a^{\prime}, b^{\prime}\right)+d\left(b^{\prime}, b\right)\right) \\
& \leq C\left[d_{1}+C(C+1) d_{2} / 2+d_{1}\right],
\end{aligned}
$$

as required by the first inequality.

If instead $a^{\prime \prime} \in \lambda_{b^{\prime}, b}$, then

$$
d(a, b) \leq d\left(a, a^{\prime}\right)+d\left(a^{\prime}, a^{\prime \prime}\right)+d\left(a^{\prime \prime}, b\right)<d_{1}+C d_{2}+d_{1}+\epsilon .
$$

and

$$
d\left(a^{\prime}, b^{\prime}\right) \leq d\left(a^{\prime}, a^{\prime \prime}\right)+d\left(a^{\prime \prime}, b^{\prime}\right)<C d_{2}+d_{1}+\epsilon .
$$

Consequently, $\operatorname{len}_{d}([a, b]) \leq 2 C d_{1}+C^{2} d_{2}+C \epsilon$ and $\operatorname{len}_{d}\left(\left[a^{\prime}, b^{\prime}\right]\right) \leq C d_{1}+$ $C^{2} d_{2}+C \epsilon$. Letting $\epsilon$ tend to 0 , the desired inequality follows.

Finally, if $a^{\prime \prime} \in[b, z]$, then $d\left(a, a^{\prime \prime}\right) \leq d\left(a, a^{\prime}\right)+d\left(a^{\prime}, a^{\prime \prime}\right) \leq d_{1}+C d_{2}$, from which it follows that

$$
\operatorname{len}_{d}([a, b]) \leq \operatorname{len}_{d}\left(\left[a, a^{\prime \prime}\right]\right) \leq C\left(d_{1}+C d_{2}\right) .
$$

Furthermore, using (5.2) again we see that $d(a, b) \leq(C+1)\left(d_{1}+\right.$ $\left.C d_{2}\right) / 2$. By $(\mathrm{GH})$ this implies that

$$
d\left(a^{\prime}, b^{\prime}\right) \leq d\left(a^{\prime}, a\right)+d(a, b)+d\left(b, b^{\prime}\right) \leq(C+5) d_{1} / 2+C(C+1) d_{2} / 2
$$

and so $2 \operatorname{len}_{d}\left(\left[a^{\prime}, b^{\prime}\right]\right) \leq C(C+5) d_{1}+C^{2}(C+1) d_{2}$.

\section{GHS implies Gromov hyperbolicity}

In this section, we aim to prove (Hyp) for GHS spaces. Our main theorem is as follows.

Theorem 6.1. Every $C$-GHS space $(X, d)$ is $K$ - $k G$-hyperbolic, where $K=$ $120 C^{11}$.

The constant $K$ is given for the convenience of the reader, but it is not optimal even for our proof (where we need $K$ to be larger than several polynomials in $C$ of degree at most 11 , so for simplicity we replaced all lower powers of $C$ by $C^{11}$ before taking a maximum).

We assume that $x, y, z \in X, w \in[x, y]$, and write $\gamma=[x, z]+[z, y]$, $A=[x, z] \cup[z, y]$. Our purpose is to prove that $w$ is at a bounded quasihyperbolic distance from $A$.

Before starting the proof let us fix some notational conventions. We push the ordering of the real line forward to $A$ via $\gamma$ and, for $x \in A, S \subset A$, we write $u \leq S$ (or $u \geq S$ ) if $u \leq v$ (or $u \geq v$, respectively) for all $v \in S$. For $\emptyset \neq S \subset A$, sup $S$ and inf $S$ are defined in the obvious way; additionally, we define $\sup \emptyset=x$ and $\inf \emptyset=y$. For any $u \in A, S_{u}$ will 
mean $S_{u}^{[x, z]}$ or $S_{u}^{[z, y]}$, depending on whether $u \leq z$ or $u>z$. We give the data $(x, y, z,[x, y],[x, z],[y, z])$ the collective name $D$.

Before we tackle the full strength version of Theorem 6.1, we shall prove a couple of weaker versions as lemmas. Our first (rather lengthy) lemma concerns the case when one of the sides of the geodesic triangle is very short.

Lemma 6.2. Let $(X, d)$ be a C-GHS space, $x, y, z \in X, w \in[x, y]$ and assume that $\operatorname{len}_{d}([z, y]) \leq d(w) / 2 C(C+1)^{2}$. Then there exists $u_{0} \in[x, z]$ such that $k\left(w, u_{0}\right) \leq 4 C^{4}(C+1)^{3}$.

Proof. We write $d_{0}=d(w) / 2 C(C+1)^{2}$, and assume for the sake of contradiction that $\operatorname{len}_{d}([z, y]) \leq d_{0}$ and $k(w,[x, z])>4 C^{4}(C+1)^{3}$. In particular, $k(x, y) \geq \log 2$, so we can apply $\left(\operatorname{Sep}_{2}\right)$ for the pair $x, y$. We start with the following:

\section{Claim.}

Let $u \in[x, z], u^{\prime} \in[x, w]$. Then

$$
d\left(u, u^{\prime}\right) \leq C(C+1) d_{0} \Rightarrow\left(S_{w} \cap[x, y]\right) \subset\left[u^{\prime}, y\right] .
$$

Let $u \in[x, z], u^{\prime} \in[w, y]$. Then

$$
d\left(u, u^{\prime}\right) \leq C(C+1) d_{0} \Rightarrow\left(S_{w} \cap[x, y]\right) \subset\left[x, u^{\prime}\right] .
$$

Proof. Suppose by contradiction that $d\left(u, u^{\prime}\right) \leq C(C+1) d_{0}$ for some $u \in[x, z]$ and $u^{\prime} \in[x, w]$, but that $u^{\prime} \in(v, y]$ for some $v \in S_{w} \cap[x, y]$. By $\left(\mathrm{Sep}_{3}\right)$ we can write

$$
k\left(u^{\prime}, w\right) \leq k(v, w) \leq \log (C+1) .
$$

By (2.8), the above inequality implies that $d\left(u^{\prime}\right) \geq d(w) /(C+1)$. This, together with

$$
d\left(u, u^{\prime}\right) \leq C(C+1) d_{0}=\frac{d(w)}{2(C+1)}
$$

and the length space assumption imply that $k\left(u, u^{\prime}\right) \leq \log 2$. This gives

$$
k(u, w) \leq \log (2 C+2)<4 C^{4}(C+1)^{3}
$$

a contradiction to the added assumption. The second implication is proven similarly.

Now we continue the proof of Lemma 6.2. Let

$$
p=\sup \left\{u \in[x, z] \mid d\left(u, u^{\prime}\right) \leq C(C+1) d_{0} \text { for some } u^{\prime} \in[x, w]\right\},
$$

and let $p^{\prime} \in[x, w]$ be such that $d\left(p, p^{\prime}\right) \leq C(C+1) d_{0}$. We choose $\lambda_{p^{\prime}, p} \in$ $\Gamma\left(p^{\prime}, p\right)$ such that $\operatorname{len}_{d}\left(\lambda_{p^{\prime}, p}\right)<C(C+2) d_{0}$, and we recall the subpath notation $\gamma[u, v]$ defined in Sect. 1. By (6.3), we see that $S_{w} \cap[x, y] \subset\left[p^{\prime}, y\right]$, 
and so by applying $\left(\operatorname{Sep}_{2}\right)$ to the path $\left[x, p^{\prime}\right]+\lambda_{p^{\prime}, p}+\gamma[p, y]$, we deduce that

$$
\operatorname{len}_{d}(\gamma[p, y])>C^{-1} d(w)-C(C+2) d_{0}>d_{0} .
$$

This implies that $p \in[x, z)$.

Next let

$$
q=\inf \left\{u \in[p, z] \mid d\left(u, u^{\prime}\right) \leq(C(C+1)-1) d_{0}\right.
$$

for some $\left.u^{\prime} \in[w, y] \cup[y, z]\right\}$.

Then $q \in[p, z]$, and there exists $q^{\prime} \in[w, y] \cup[y, z]$ such that we have the inequality $d\left(q, q^{\prime}\right) \leq(C(C+1)-1) d_{0}$.

Consequently, there exists $q^{\prime \prime} \in[w, y]$ such that $d\left(q, q^{\prime \prime}\right)<\left(C^{2}+C\right) d_{0}$. We choose $\lambda_{q, q^{\prime \prime}} \in \Gamma\left(q, q^{\prime \prime}\right)$ such that $\operatorname{len}_{d}\left(\lambda_{q, q^{\prime \prime}}\right) \leq\left(C^{2}+C\right) d_{0}$.

By (6.3) and (6.4), we see that $\left(S_{w} \cap[x, y]\right) \subset\left[p^{\prime}, q^{\prime \prime}\right]$, and so by applying $\left(\mathrm{Sep}_{2}\right)$ to the path

$$
\left[x, p^{\prime}\right]+\lambda_{p^{\prime}, p}+[p, q]+\lambda_{q, q^{\prime \prime}}+\left[q^{\prime \prime}, y\right],
$$

we deduce that $\operatorname{len}_{d}\left(I_{w}\right)>C^{-1} d(w)-2 C(C+1) d_{0}=d(w) / C(C+1)$, where $I_{w} \equiv\left\{u \in S_{w} \mid p<u<q\right\}$.

Let us fix $u \in I_{w}$. By the extremality of $p$ and $q$ we have

$$
d(u,[y, z] \cup[w, y])>(C(C+1)-1) d_{0},
$$

and

$$
d(u,[x, w])>C(C+1) d_{0} .
$$

Since $S_{u}$ surely intersects $[x, y] \cup[y, z]$, by (Sep) we must have $d(u)>C d_{0}$.

Since $k(w,[x, z])>4 C^{4}(C+1)^{3}$ and $\operatorname{len}_{d}([u, w]) \leq C d(u, w) \leq$ $C^{2} d(w)$, there must be a point $r \in[w, u]$ with

$$
d(r)<d(w) / 4 C^{2}(C+1)^{3}=d_{0} / 2 C(C+1) .
$$

There exist points $r^{\prime} \in[w, x] \cup[x, u]$ and $r^{\prime \prime} \in[w, y] \cup[y, z] \cup[z, u]$ which are also in $S_{r}^{[w, u]}$, and so strictly within a distance $d_{0} /(C+1)$ of each other.

Suppose first that $r^{\prime} \in[w, x]$. Since $d\left(r^{\prime}, r^{\prime \prime}\right)<d_{0} /(C+1)$ the maximality of $p$ implies that we cannot have $r^{\prime \prime} \in[u, z] \cup[z, y]$. We shall now show that $r^{\prime \prime} \in[w, y]$ leads also to a contradiction. To do so observe first that $d\left(r^{\prime}\right) \vee d\left(r^{\prime \prime}\right) \leq(C+1) d(r)<d_{0} / 2 C$. Assuming $r^{\prime \prime} \in[w, y]$ leads to

$$
\begin{aligned}
\operatorname{len}_{d}\left(\left[r^{\prime}, r^{\prime \prime}\right]\right) & =\operatorname{len}_{d}\left(\left[r^{\prime}, w\right]\right)+\operatorname{len}_{d}\left(\left[w, r^{\prime \prime}\right]\right) \\
& \geq 2\left[d(w)-\left(d\left(r^{\prime}\right) \vee d\left(r^{\prime \prime}\right)\right)\right] \\
& \geq 2\left[d(w)-\frac{d_{0}}{2 C}\right]>d(w)>C d\left(r^{\prime}, r^{\prime \prime}\right),
\end{aligned}
$$

which contradicts $(\mathrm{GH})$. 
Thus we must have $r^{\prime} \in[x, u]$. If $r^{\prime \prime} \in[u, z]$, then

$$
\operatorname{len}_{d}\left(\left[r^{\prime}, r^{\prime \prime}\right]\right) \geq d(u)-d\left(r^{\prime}\right) \geq C d_{0}-d_{0} / 2 C>C d\left(r^{\prime}, r^{\prime \prime}\right),
$$

which contradicts again $(\mathrm{GH})$.

If $r^{\prime \prime} \in[z, y]$, then

$$
d\left(r^{\prime}, z\right) \leq d\left(r^{\prime}, r^{\prime \prime}\right)+d_{0}<(C+2) d_{0} /(C+1) .
$$

On the other hand by the minimality of $q$ we have $\operatorname{len}_{d}\left(\left[r^{\prime}, u\right]\right) \geq C d_{0}-$ $d_{0} /(2 C)$ and

$$
\operatorname{len}_{d}([u, z]) \geq \operatorname{len}_{d}([q, z]) \geq d(q, z) \geq[C(C+1)-1] d_{0} .
$$

Thus

$$
\begin{aligned}
\operatorname{len}_{d}\left(\left[r^{\prime}, z\right]\right) \geq[C(C+2)-1-1 /(2 C)] d_{0} & \geq[C(C+2) /(C+1)] d_{0} \\
& >C\left[d\left(r^{\prime}, r^{\prime \prime}\right)+d_{0}\right] \\
& \geq C d\left(r^{\prime}, z\right),
\end{aligned}
$$

again contradicting $(\mathrm{GH})$.

In conclusion we must have $r^{\prime \prime} \in[y, w]$. Since $d\left(r^{\prime}, r^{\prime \prime}\right)<\frac{d_{0}}{C+1}$ and $r^{\prime} \leq u<q$, the minimality of $q$ implies that $r^{\prime}<p$. We shall now apply the first estimate from Lemma 5.3 with the choice of data

$$
\left(a, a^{\prime}, b, b^{\prime}, d_{1}, d_{2}\right)=\left(r^{\prime \prime}, r^{\prime}, p^{\prime}, p, d\left(r^{\prime \prime}, r^{\prime}\right) \vee d\left(p^{\prime}, p\right), d\left(r^{\prime}\right)\right) .
$$

Since $d\left(r^{\prime}, r^{\prime \prime}\right) \vee d\left(p^{\prime}, p\right) \leq C(C+1) d_{0}$ and $d\left(r^{\prime}\right) \leq d_{0} /(2 C)$, we obtain

$$
\begin{aligned}
\operatorname{len}_{d}\left(\left[r^{\prime \prime}, p^{\prime}\right]\right) & =\operatorname{len}_{d}([a, b]) \leq 2 C d_{1}+\frac{C^{2}(C+1)}{2} d_{2} \\
& \leq 2 C^{2}(C+1) d_{0}+\frac{C(C+1)}{4} d_{0} \\
& =\left[2 C^{3}+(9 / 4) C^{2}+C / 4\right] d_{0} .
\end{aligned}
$$

This gives a contradiction, since

$$
\begin{aligned}
\operatorname{len}_{d}\left(\left[r^{\prime \prime}, p^{\prime}\right]\right) & \geq \operatorname{len}_{d}\left(\left[r^{\prime \prime}, w\right]\right) \geq d(w)-d\left(r^{\prime \prime}\right)>d(w)-d_{0} / 2 C= \\
& =\left[2 C^{3}+4 C^{2}+2 C-1 /(2 C)\right] d_{0} .
\end{aligned}
$$

The proof of the lemma is completed.

For $0<\eta \leq \epsilon$, we define an $(\epsilon, \eta)$-shortcut set for our data $D$ to be a collection of points $P=\left\{p_{m}, p_{m}^{\prime}\right\}_{m=0}^{m_{0}}, m_{0} \in \mathbb{N}$, that satisfy the following five properties:

(i) $p_{0} \in[x, w], p_{0}^{\prime} \in A, p_{m_{0}} \in A, p_{m_{0}}^{\prime} \in[w, y]$;

(ii) $p_{m}, p_{m}^{\prime} \in \gamma$, for all $0<m<m_{0}$;

(iii) $p_{m-1}^{\prime} \leq p_{m} \leq p_{m}^{\prime} \leq p_{m+1}$ for all $0<m<m_{0}$; 
(iv) $\sum_{m=0}^{m_{0}} d\left(p_{m}, p_{m}^{\prime}\right)<\epsilon$;

(v) $d\left(p_{m}, p_{m}^{\prime}\right) \leq \eta$, for all $0 \leq m \leq m_{0}$.

Given such a set $P$, we write $A_{P}=\bigcup_{m=1}^{m_{0}}\left(p_{m-1}^{\prime}, p_{m}\right), A_{P}^{\prime}=S_{w}^{[x, y]} \cap A_{P}$, and $A_{P}^{\prime \prime}=S_{w}^{[x, y]} \cap\left(\left[x, p_{0}\right] \cup\left[p_{m_{0}}^{\prime}, y\right]\right)$. We use shortcut sets to travel from $x$ to $y$ by connecting bits of geodesics with shortcut paths from $p_{m}$ to $p_{m}^{\prime}$. If $\epsilon$ is small, we can then use $\left(\operatorname{Sep}_{2}\right)$ to get a lower bound for $\operatorname{len}_{d}\left(A_{P}^{\prime}\right)$; in particular $A_{P}^{\prime}$ is non-empty in that case. In fact, we have the following technical lemma.

Lemma 6.5. Suppose that $(X, d)$ is a C-GHS space, $x, y, z \in X, w \in$ $[x, y]$ and that $P$ is an $\left(L_{0}, L_{0} / 2\right)$-shortcut set for the data $D$, where $L_{0}=$ $2 d(w) / 3 C$. Suppose also that $k(u, w) \geq \log (3 C+3)$ for all $u \in A$. Then $A_{P}^{\prime \prime}=\emptyset$ and $\operatorname{len}_{d}\left(A_{P}^{\prime}\right) \geq d(w) / 3 C>0$.

Proof. By hypothesis, there are paths $\lambda_{m} \in \Gamma\left(p_{m}, p_{m}^{\prime}\right)$ such that $\sum_{m=0}^{m_{0}} \operatorname{len}_{d}\left(\lambda_{m}\right) \leq L_{0}$. We define the joined path

$$
\lambda_{P}=\left[x, p_{0}\right]+\lambda_{0}+\sum_{m=1}^{m_{0}}\left(\left[p_{m-1}^{\prime}, p_{m}\right]+\lambda_{m}\right)+\left[p_{m_{0}}^{\prime}, y\right] .
$$

Applying $\left(\operatorname{Sep}_{2}\right)$ to $\lambda_{P}$, we see that $\operatorname{len}_{d}\left(A_{P}^{\prime}\right)+\operatorname{len}_{d}\left(A_{P}^{\prime \prime}\right) \geq C^{-1} d(w)-L_{0}$ $=L_{0} / 2>0$.

To show that $A_{P}^{\prime \prime}=\varnothing$ assume by contradiction that there is a point $p \in\left[x, p_{0}\right]$ such that $p \in S_{w}^{[x, y]}$. Using $\left(\operatorname{Sep}_{3}\right)$, we see that

$$
k\left(p_{0}, w\right) \leq k(p, w) \leq \log (C+1),
$$

which implies in particular that $d\left(p_{0}\right) \geq d(w) /(C+1)$. Because $d\left(p_{0}, p_{0}^{\prime}\right) \leq$ $d(w) / 3 C$, it follows from the length space assumption that $k\left(p_{0}, p_{0}^{\prime}\right) \leq$ $\log 3$. By the triangle inequality, we get $k\left(p_{0}^{\prime}, w\right) \leq \log 3(C+1)$, contradicting the hypothesis.

Similarly, we must have $S_{w}^{[x, y]} \cap\left[p_{m_{0}}^{\prime}, y\right]=\emptyset$. Thus $A_{P}^{\prime \prime}=\emptyset$, and consequently $\operatorname{len}_{d}\left(A_{P}^{\prime}\right) \geq L_{0} / 2$, as desired.

We now come to our main lemma concerning shortcut sets. We are really interested in applying this lemma only in the cases $m_{0}=1$ and $m_{0}=2$, but stating it for arbitrary $m_{0}$ allows us to write down a proof simultaneously for both cases. Note that, since we are aiming to prove Gromov hyperbolicity, the assumption $k(u, w) \geq \log (3 C+3)$ does not make the lemma any less useful.

Lemma 6.6. Suppose that $(X, d)$ is a $C$-GHS space, and $x, y, z \in X, w \in$ $[x, y]$. Let $\epsilon=d(w) / 3 C$ and and $\eta=\epsilon / C_{1}$, where $C_{1}=\left(C^{3}+3 C^{2}+\right.$ $6 C+6) / 2$. Suppose that $P$ is an $(\epsilon, \eta)$-shortcut set for the data $D$, and $k(u, w) \geq \log (3 C+3)$ for all $u \in A$. Then there exists a point $u \in A_{P}^{\prime}$ such that $d(u, w) \leq C d(w)$ and $d(u) \geq \eta / C$. 
Proof. By the previous lemma the set $A_{P}^{\prime}$ is not empty. Since $A_{P}^{\prime}$ is a subset of $S_{w}$, for $u \in A_{P}^{\prime}$ we have $d(u, w) \leq C d(w)$. For the purpose of contradiction let us assume that $d(u)<\eta / C$ for all $u \in A_{P}^{\prime}$.

We first wish to modify $P$ to define a new shortcut set $Q$ which avoids as much of the initial and final parts of $\gamma$ as possible. Let

$$
a_{-}^{\prime}=\sup \left(\left\{p_{0}^{\prime}\right\} \cup\left\{u \in A_{P}^{\prime} \mid S_{u} \cap[x, w] \neq \emptyset\right\}\right) \in \overline{A_{P}^{\prime}} .
$$

We define $a_{-}$by the rule: $a_{-}=p_{0}$ if $a_{-}^{\prime}=p_{0}^{\prime}$, and otherwise let $a_{-}$be any point on $[x, w]$ for which $d\left(a_{-}^{\prime}, a_{-}\right)<\eta$; this last choice is possible because $d\left(u, u^{\prime}\right) \leq C d(u) \leq \eta$ whenever $u \in A_{P}^{\prime}, u^{\prime} \in S_{u} \cap[x, w]$. Next we set

$$
a_{+}=\inf \left(\left\{p_{m_{0}}\right\} \cup\left\{u \in A_{P}^{\prime} \mid u \geq a_{-}^{\prime}, S_{u} \cap[w, y] \neq \emptyset\right\}\right) \in \overline{A_{P}^{\prime}} .
$$

We also define $a_{+}^{\prime}$ by the rule $a_{+}^{\prime}=p_{m_{0}}^{\prime}$ if $a_{+}=p_{m_{0}}$, and otherwise $a_{+}^{\prime}$ is any point on $[w, y]$ for which $d\left(a_{+}^{\prime}, a_{+}\right)<\eta$; this last choice is possible for similar reasons to those for $a_{-}$. Note that $d\left(a_{-}^{\prime}\right) \leq \eta / C$ if $a_{-}^{\prime} \neq p_{0}^{\prime}$, and that $d\left(a_{+}\right) \leq \eta / C$ if $a_{+} \neq p_{m_{0}}$. In this case we have

$$
d\left(a_{-}, a_{-}^{\prime}\right) \leq C d\left(a_{-}^{\prime}\right) \leq \eta, d\left(a_{+}, a_{+}^{\prime}\right) \leq C d\left(a_{+}\right) \leq \eta .
$$

We now define the new shortcut set $Q=\left\{q_{m}, q_{m}^{\prime}\right\}_{m=0}^{m_{1}}$ by replacing $p_{0}$, $p_{0}^{\prime}, p_{m_{0}}$, and $p_{m_{0}}^{\prime}$ by $a_{-}, a_{-}^{\prime}, a_{+}$, and $a_{+}^{\prime}$, respectively, and also discarding any pairs $p_{m}, p_{m}^{\prime}$ which are not elements of $\left[a_{-}^{\prime}, a_{+}\right]$(i.e. we keep only those pairs $\left(p_{m}, p_{m}^{\prime}\right)$ for which $\left.a_{-}^{\prime} \leq p_{m} \leq p_{m}^{\prime} \leq a_{+}\right)$.

Now $Q$ is an $(\epsilon+2 \eta, \eta)$-shortcut set and, since $\epsilon+2 \eta<2 \epsilon$, Lemma 6.5 implies that $\operatorname{len}_{d}\left(A_{Q}^{\prime}\right) \geq \epsilon>0$. Note also that $A_{Q}^{\prime} \subset A_{P}^{\prime}$. By symmetry of $x$ and $y$, we may assume that $A_{Q}^{\prime} \cap[x, z]$ is non-empty. Applying symmetry may seem dangerous since $a_{-}^{\prime}$ satisfies a stronger extremality property than $a_{+}$, but the only fact following from the extremality of these points that we use below is that $S_{u}$ cannot intersect $[x, y]$ if $a_{-}^{\prime}<u<a_{+}$, and this can safely be deduced whether or not we swap the roles of $x$ and $y$.

Letting

$$
\begin{aligned}
T_{1}=\left\{u \in\left[a_{-}^{\prime}, z\right] \mid d(u) \leq(C+1) \eta / C, \exists u^{\prime} \in[z, y]:\right. \\
\\
\left.d\left(u, u^{\prime}\right) \leq \eta, d\left(u^{\prime}\right) \leq(C+1) \eta / C\right\},
\end{aligned}
$$

we claim that $A_{Q}^{\prime} \cap[x, z] \subset T_{1}$. First observe that for $u \in A_{Q}^{\prime} \backslash\left\{a_{-}^{\prime}\right\}$ we have $u>a_{-}^{\prime}$ which implies that $S_{u} \cap[x, w]=\emptyset$. Similarly, for $u \in A_{Q}^{\prime} \backslash\left\{a_{+}\right\}$we have $u<a_{+}$and so $S_{u} \cap[w, y]=\emptyset$. We conclude that $S_{u} \cap[x, y] \subset\left\{a_{-}^{\prime}, a_{+}\right\}$ for all $u \in A_{Q}^{\prime}$. Thus by applying $\left(\mathrm{Sep}_{2}\right)$ for the slice $S_{u}, u \in A_{Q}^{\prime} \cap[x, z]$, to the path $[x, y]+[y, z]$, we get a point $u^{\prime} \in S_{u} \cap[z, y]$, and our claim readily follows. Defining $a=\inf T_{1}$, it follows that $a \leq A_{Q}^{\prime}$.

Note also that $d(a) \leq(C+1) \eta / C$, and there exists $a^{\prime} \in[z, y]$ with $d\left(a, a^{\prime}\right) \leq \eta$ and $d\left(a^{\prime}\right) \leq(C+1) \eta / C$. We distinguish two cases. Either $a^{\prime} \in A_{Q}$, or $q_{m}<a^{\prime}<q_{m}^{\prime}$ for some $1 \leq m \leq m_{1}$. In the first case, we define $a^{\prime \prime}=a^{\prime}$, and in the second case we let $a^{\prime \prime}=q_{m}^{\prime}$. 
We form a new shortcut set $R$ from $Q$ by inserting the pair $a, a^{\prime \prime}$ and discarding any pairs in $Q$ that are contained in $\left[a, a^{\prime \prime}\right]$. We claim that $d\left(a, a^{\prime \prime}\right) \leq \epsilon-2 \eta$.

The claim is easy to see if $a^{\prime \prime}=a^{\prime}$ since then

$$
d\left(a, a^{\prime \prime}\right)=d\left(a, a^{\prime}\right) \leq \eta \leq \epsilon-2 \eta
$$

by the choice of the magnitude of $\epsilon$ and $\eta$ from the statement.

If $a^{\prime \prime}=q_{m}^{\prime}$ we consider two cases: $q_{m} \geq z$ and $q_{m}<z$. In the first case the points $z \leq q_{m}<a^{\prime}<q_{m}^{\prime}=a^{\prime \prime}$ are situated on $[z, y]$. By applying (GH), we obtain

$$
d\left(a^{\prime}, a^{\prime \prime}\right) \leq \operatorname{len}_{d}\left(\left[a^{\prime}, a^{\prime \prime}\right]\right) \leq \operatorname{len}_{d}\left(\left[q_{m}, q_{m}^{\prime}\right]\right) \leq C \eta,
$$

which implies that

$$
d\left(a, a^{\prime \prime}\right) \leq d\left(a, a^{\prime}\right)+d\left(a^{\prime}, a^{\prime \prime}\right) \leq \eta+C \eta \leq \epsilon-2 \eta .
$$

Consider the second case $q_{m}<z$. Since $a \leq A_{Q}^{\prime}$, and $A_{Q}^{\prime} \cap[x, z]$ is a non-empty subset of $\left[x, q_{m}\right]$, we must have $a<q_{m}$. Thus we may apply the second conclusion of Lemma 5.3 with data

$$
\left(a, a^{\prime}, b, b^{\prime}, d_{1}, d_{2}\right)=\left(a, a^{\prime}, q_{m}, q_{m}^{\prime}, \eta,(C+1) \eta / C\right)
$$

to deduce that

$$
\begin{aligned}
d\left(a, a^{\prime \prime}\right)=d\left(a, q_{m}^{\prime}\right) & \leq d\left(a, a^{\prime}\right)+\operatorname{len}_{d}\left(\left[a^{\prime}, q_{m}^{\prime}\right]\right) \\
& \leq \eta\left[1+C(C+5) / 2+C(C+1)^{2} / 2\right] \\
& =\eta\left[C^{3}+3 C^{2}+6 C+2\right] / 2 \leq \epsilon-2 \eta .
\end{aligned}
$$

Our claim follows. Since $Q$ is an $(\epsilon+2 \eta, \eta)$ shortcut set, it follows that $R$ is a $(2 \epsilon, \epsilon)$-shortcut set. Thus, using Lemma $6.5, \operatorname{len}_{d}\left(A_{R}^{\prime}\right) \geq \epsilon>0$.

Since $a \leq A_{Q}^{\prime}$ and $A_{R}^{\prime} \subset A_{Q}^{\prime}$, we have $a \leq A_{R}^{\prime}$. On the other hand,

$$
A_{R}^{\prime} \cap[x, z] \subset A_{R} \cap[x, z] \subset\left(a_{-}^{\prime}, a\right) .
$$

Thus $A_{R}^{\prime} \cap[x, z]=\emptyset$ and so $A_{R}^{\prime} \subset A_{R} \cap[z, y] \subset\left(a^{\prime \prime}, a_{+}\right)$. We choose $b^{\prime} \in A_{R}^{\prime}$ such that $0<\operatorname{len}_{d}\left(A_{R}^{\prime} \cap\left(b^{\prime}, a_{+}\right)\right)<\eta$.

Let $\lambda_{a_{-}, a_{-}^{\prime}}$ be a path from $a_{-}$to $a_{-}^{\prime}$ such that $\operatorname{len}_{d}\left(\lambda_{a_{-}, a_{-}^{\prime}}\right)<2 \eta$. By $\left(\mathrm{Sep}_{2}\right)$, there must be some point $b$ in $S_{b^{\prime}}^{[y, z]}$ that also lies on the path $\left[y, a_{-}\right]+$ $\lambda_{a_{-}, a_{-}^{\prime}}+\left[a_{-}, z\right]$. But we cannot have $b \in\left[y, a_{-}\right]$by the extremality of $a_{-}^{\prime}$

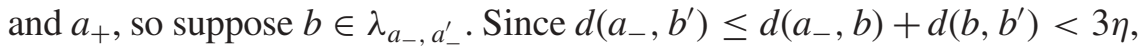
we can define a new shortcut set $U$ from $Q$ by inserting the initial pair $a_{-}, b^{\prime}$ and discarding all other pairs except those that lie in $\left[b^{\prime}, y\right] \cup\left\{a_{+}^{\prime}\right\}$. Since $Q$ is an $(\epsilon+2 \eta, \eta)$-shortcut set, it follows that $U$ is an $(\epsilon+5 \eta, \eta)$-shortcut set, and so a $(2 \epsilon, \epsilon)$-shortcut set. Thus, as usual, $\operatorname{len}_{d}\left(A_{U}^{\prime}\right)>\epsilon>0$. Since the collection of pairs in $U$ other than $a_{-}, b^{\prime}$ coincide with the collection of pairs $u, u^{\prime}$ in $R$ for which $u>b$, it is clear that $A_{U}^{\prime}=A_{R}^{\prime} \cap\left(b^{\prime}, a_{+}\right)$. Thus $\operatorname{len}_{d}\left(A_{U}^{\prime}\right)<\eta<\epsilon$, giving us a contradiction. 
Finally, we consider the case where $b \in\left(a_{-}^{\prime}, z\right]$. By minimality of $a$, we must have $b \geq a$. Since $b^{\prime} \in A_{R}^{\prime} \subset A_{Q}^{\prime}$, we have $d\left(b^{\prime}\right) \leq \eta / C, d\left(b, b^{\prime}\right) \leq \eta$, and $d(b) \leq(C+1) \eta / C$. Thus $b \geq a$. Now $d\left(a, a^{\prime}\right) \vee d\left(b, b^{\prime}\right) \leq \eta, d\left(a^{\prime}\right)<$ $(C+1) \eta / C$, and

$$
\operatorname{len}_{d}\left(\left[a^{\prime}, b^{\prime}\right]\right) \geq \operatorname{len}_{d}\left(\left[a^{\prime \prime}, b^{\prime}\right]\right) \geq \operatorname{len}_{d}\left(A_{R}^{\prime} \cap\left(a^{\prime \prime}, b^{\prime}\right)\right) \geq \epsilon-\eta,
$$

which contradicts Lemma 5.3, so we are done.

We are now ready to tackle Theorem 6.1.

Proof of Theorem 6.1. We set $d_{0}=2 C^{3} d(w) / K$ and let $e^{\prime}=\sup T_{1}$, where

$$
\begin{aligned}
T_{1}=\{x\} \cup\left\{u \in A \mid d(u) \leq d_{0},\right. & \exists u^{\prime} \in[x, w]: \\
d\left(u, u^{\prime}\right) & \left.\leq d_{0} \text { and } d\left(u^{\prime}\right) \leq d_{0}\right\} .
\end{aligned}
$$

If $e^{\prime}=x$, let $e=x$ also. If $e^{\prime} \neq x$, then $d\left(e^{\prime}\right) \leq d_{0}$, and we may choose $e \in[x, w]$ and a path $\lambda_{e, e^{\prime}} \in \Gamma\left(e, e^{\prime}\right)$ such that $d\left(e, e^{\prime}\right) \leq d_{0}, d(e) \leq d_{0}$, and $\operatorname{len}_{d}\left(\lambda_{e, e^{\prime}}\right) \leq 2 d_{0}$. If $e^{\prime}>z$ then, using the fact that $4 C^{5}(C+1)^{2}<K$, it follows that

$$
\operatorname{len}_{d}\left(\left[e, e^{\prime}\right]\right) \leq C d\left(e, e^{\prime}\right)<d(w) / 2 C(C+1)^{2},
$$

and we can apply Lemma 6.2 to the triangle $e e^{\prime} y$. We deduce that

$$
k(w, A) \leq k\left(w,\left[e^{\prime}, y\right]\right) \leq 4 C^{4}(C+1)^{3}<K,
$$

and the theorem follows. We may therefore assume that $e^{\prime} \leq z$.

Next we let $f=\inf T_{2}$, where

$$
\begin{array}{r}
T_{2}=\{z\} \cup\left\{u \in[x, z] \mid d(u) \leq d_{0}, \exists u^{\prime} \in[z, y]:\right. \\
\left.d\left(u, u^{\prime}\right) \leq d_{0} \text { and } d\left(u^{\prime}\right) \leq d_{0}\right\} .
\end{array}
$$

If $f=z$, let $f^{\prime}=z$. Otherwise, $d(f) \leq d_{0}$ and we find $f^{\prime} \in[z, y]$ such that $d\left(f, f^{\prime}\right) \leq d_{0}$ and $d\left(f^{\prime}\right) \leq d_{0}$.

There are two cases to consider. Suppose first that $f<e^{\prime}$ (and so in particular, $f \neq z$ and $e^{\prime} \neq x$ ). In this case we argue again using Lemma 6.2 to a smaller geodesic triangle containing $w$ in one of its sides. To do that let us assume first that $\operatorname{len}_{d}\left(\left[e^{\prime}, f\right]\right)>C^{2} d_{0}$. Then $(\mathrm{GH})$ implies that

$$
d\left(\left[e^{\prime}, z\right], f\right) \geq \operatorname{len}_{d}\left(\left[e^{\prime}, f\right]\right) / C \geq C d_{0} .
$$

Since $d(f) \leq d_{0}$ we have $S_{f}^{[x, z]} \subset B_{d}\left(f, C d_{0}\right)$ which implies that $\left[e^{\prime}, z\right] \cap$ $S_{f}^{[x, z]}=\emptyset$. Applying $\left(\operatorname{Sep}_{2}\right)$ to the path $[x, e]+\lambda_{e, e^{\prime}}+\left[e^{\prime}, z\right]$, we deduce that there exists $u \in S_{f}^{[x, z]} \cap\left([x, e]+\lambda_{e, e^{\prime}}\right)$. Let $f^{\prime \prime}=u$ if $u \in[x, e]$, and otherwise let $f^{\prime \prime}=e$. In the first case, note that

$$
d\left(f^{\prime \prime}, f^{\prime}\right)<d\left(f^{\prime \prime}, f\right)+d\left(f, f^{\prime}\right) \leq C d_{0}+d_{0} .
$$


In the second case we have

$$
d\left(f^{\prime \prime}, f^{\prime}\right)<\operatorname{len}_{d}\left(\lambda_{e, e^{\prime}}\right)+C d_{0}+d_{0} \leq(C+3) d_{0} .
$$

Alternatively assume that $\operatorname{len}_{d}\left(\left[e^{\prime}, f\right]\right)<C^{2} d_{0}$ (recall still $\left.f<e^{\prime}\right)$. Let $f^{\prime \prime}=e$ and note that

$$
d\left(f^{\prime \prime}, f^{\prime}\right) \leq d\left(f^{\prime \prime}, e^{\prime}\right)+d\left(e^{\prime}, f\right)+d\left(f, f^{\prime}\right)<d_{0}\left[1+C^{2}+1\right] .
$$

By Gehring-Hayman, we conclude in either case that $w$ lies in a triangle $f^{\prime \prime} f^{\prime} y$ with the property that $\operatorname{len}_{d}\left(\left[f^{\prime}, f^{\prime \prime}\right]\right)<\left(C^{3}+3 C\right) d_{0}$. Since $\left(C^{3}+\right.$ $3 C) d_{0}<d(w) / 2 C(C+1)^{2}$, the result then follows by Lemma 6.2.

We may now turn to the more difficult situation when $f \geq e^{\prime}$.

Next we define $g=\inf T_{3}$, where

$$
\begin{aligned}
T_{3}=\{y\} \cup\left\{u \in A \mid d(u) \leq d_{0}, \quad \exists u^{\prime} \in[w, y]:\right. & \\
d\left(u, u^{\prime}\right) & \left.\leq d_{0} \text { and } d\left(u^{\prime}\right) \leq d_{0}\right\} .
\end{aligned}
$$

If $g=y$, let $g^{\prime}=y$ also. If $g \neq y$, then $d(g) \leq d_{0}$, and we may choose $g^{\prime} \in[w, y]$ and a path $\lambda_{g, g^{\prime}} \in \Gamma\left(g, g^{\prime}\right)$ such that $d\left(g, g^{\prime}\right) \leq d_{0}, d\left(g^{\prime}\right) \leq d_{0}$, and $\operatorname{len}_{d}\left(\lambda_{g, g^{\prime}}\right) \leq 2 d_{0}$. We also define $h=\sup T_{4}$, where

$$
\begin{array}{r}
T_{4}=\{z\} \cup\left\{u \in[z, y] \mid d(u) \leq d_{0}, \exists u^{\prime} \in[x, z]:\right. \\
\left.d\left(u, u^{\prime}\right) \leq d_{0} \text { and } d\left(u^{\prime}\right) \leq d_{0}\right\} .
\end{array}
$$

As before, we may assume without loss of generality that $h \leq g$, and so $f^{\prime} \leq h \leq g$.

We now assume for the sake of contradiction that $k(w, A)>K$. Letting $P=\left\{a_{m}, a_{m}^{\prime} \mid m=0,1,2\right\}$, where $\left(a_{0}, a_{0}^{\prime}, a_{1}, a_{1}^{\prime}, a_{2}, a_{2}^{\prime}\right)=$ $\left(e, e^{\prime}, f, f^{\prime}, g, g^{\prime}\right)$, we see that $P$ is an $(\epsilon, \eta)$-shortcut set for $\epsilon=d(w) / 3 C$, $\eta=d(w) / 3 C C_{1} \geq d_{0}$. Note that we are including the possibility of degenerate shortcut sets such as $\left(e, e^{\prime}, f, f^{\prime}, g, g^{\prime}\right)=(x, x, z, z, y, y)$. By Lemma 6.6, there exists $v \in A_{P}^{\prime}=\left(e^{\prime}, f\right) \cup\left(f^{\prime}, g\right)$ such that $d(v, w) \leq$ $C d(w)$ and $d(v) \geq d(w) / 3 C^{2} C_{1}=K d_{0} / 6 C^{5} C_{1}>5 C^{3} d_{0} / 2$.

Consider first the case $v \in\left(e^{\prime}, f\right)$. Since $k(v, w)>K$ and $\operatorname{len}_{d}([v, w]) \leq$ $C^{2} d(w)$, it follows that there exists a point $p \in[v, w]$ such that $d(p)<$ $C^{2} d(w) / K=d_{0} / 2 C$. Now $S_{p}^{[v, w]}$ must intersect $[w, x] \cup[x, v]$ at some point $p^{\prime}$ and $[v, z] \cup[z, y] \cup[y, w]$ at some point $p^{\prime \prime}$; note that $d\left(p^{\prime}\right) \vee$ $d\left(p^{\prime \prime}\right) \vee d\left(p^{\prime}, p^{\prime \prime}\right)<d_{0}$. If $p^{\prime} \in[x, w]$, we cannot choose the point $p^{\prime \prime}$ with the desired properties. In fact, $p^{\prime \prime} \in[v, z]$ contradicts the maximality of $e^{\prime}$, while if $p^{\prime \prime} \in[z, y]$, then applying Lemma 6.2 to the triangle $p^{\prime} p^{\prime \prime} y$ contradicts the lower bound for $k(w, A)$. Finally if $p^{\prime \prime} \in[w, y]$, then

$$
\operatorname{len}_{d}\left(\left[p^{\prime}, p^{\prime \prime}\right]\right) \geq d(w)-d\left(p^{\prime}\right)>C d_{0} \geq C d\left(p^{\prime}, p^{\prime \prime}\right),
$$

contradicting $(\mathrm{GH})$.

Consequently, we must have $p^{\prime} \in[x, v]$. Now $p^{\prime \prime} \notin[y, w]$ since then applying Lemma 6.2 to the triangle $p^{\prime} p^{\prime \prime} x$ contradicts the lower bound 
for $k(w, A)$, and $p^{\prime \prime} \notin[v, z]$ since then $\operatorname{len}\left(\left[p^{\prime}, p^{\prime \prime}\right]\right) \geq d(v)-d\left(p^{\prime}\right)>$ $C d\left(p^{\prime}, p^{\prime \prime}\right)$. Finally if $p^{\prime \prime} \in[z, y]$, then we get a contradiction to the minimality of $f$.

If instead $v \in\left(f^{\prime}, g\right)$, we find, as before, points $p^{\prime} \in[w, y] \cup[y, v]$ and $p^{\prime \prime} \in[v, z] \cup[z, x] \cup[x, w]$ such that $d\left(p^{\prime}\right) \vee d\left(p^{\prime \prime}\right) \vee d\left(p^{\prime}, p^{\prime \prime}\right)<d_{0}$. All possibilities are then ruled out as before with the exception that there is no extremality of $f^{\prime}$ to rule out the possibility that $p^{\prime} \in[y, v], p^{\prime \prime} \in[x, z]$. However, the minimality of $f$ ensures that $p^{\prime \prime} \geq f$. We can therefore apply Lemma 5.3 to the data

$$
\left(a, a^{\prime}, b, b^{\prime}, d_{1}, d_{2}\right)=\left(f^{\prime}, f, p^{\prime}, p^{\prime \prime}, d_{0}, d_{0}\right)
$$

(with the roles of $x, y, z$ switched among themselves) to deduce that

$$
\operatorname{len}_{d}\left(\left[f^{\prime}, p^{\prime}\right]\right) \leq\left(2 C+C^{2}(C+1) / 2\right) d_{0} \leq 3 C^{3} d_{0},
$$

which contradicts the fact that

$$
\operatorname{len}_{d}\left(\left[f^{\prime}, p^{\prime}\right]\right) \geq 2 d(v)-d\left(p^{\prime}\right)-d\left(f^{\prime}\right)>3 C^{3} d_{0} .
$$

\section{Appendix: Background on Loewner spaces}

In this appendix, we define some basic concepts related to the modulus, and develop some properties of Loewner spaces.

First a metric measure space $(X, d, \mu)$ is simply a metric space with a Borel measure $\mu$ attached. If additionally $(X, d)$ is rectifiably connected, we define $A(\Gamma)$, the set of $\Gamma$-admissable weights for a family $\Gamma$ of paths in $X$, to be the set of all Borel functions $\rho: X \rightarrow[0, \infty]$ such that $\int_{\gamma} \rho|d z| \geq 1$ for each path $\gamma \in \Gamma$. For $Q \geq 1$, we then define the $Q$-modulus of $\Gamma$ as

$$
\bmod _{Q}(\Gamma)=\inf _{\rho \in A(\Gamma)} \int_{X} \rho^{Q} d \mu .
$$

When $S \subseteq X$ is a domain in $X$ and $E, F$ are two disjoint compacta in $S$ we denote by $\Gamma(E, F ; S)$ the family of paths contained in $S$ that begin in $E$ and terminate in $F$. In this case we define $A(E, F ; S)=A(\Gamma(E, F ; S))$ and $\bmod _{Q}(E, F ; S)=\bmod _{Q}(\Gamma(E, F ; S))$; we omit $S$ in this notation when $S=X$. We define $\Gamma(E, F ; S)=\infty$ if $E$ and $F$ overlap.

Suppose $(X, d, \mu)$ is a metric measure space. Given $Q \geq 1$, we say that $X$ is $Q$-regular if there exists a constant $C>0$ such that

$$
\forall x \in X, 0<r \leq \operatorname{dia}_{d}(X): \quad C^{-1} r^{Q} \leq \mu\left(B_{d}(x, r)\right) \leq C r^{Q} .
$$

If only the upper bound above holds, we say that $X$ is upper $Q$-regular. We denote the smallest such constant $C$ for which the (upper) $Q$-regularity condition holds by $C(\mu, Q)$ (or $C^{+}(\mu, Q)$, respectively). We define local 
$Q$-regularity in a similar fashion except that the upper and lower bounds for $\mu\left(B_{d}(x, r)\right)$ apply only when $r<d(x) / 2$.

Recall that

$$
\Delta_{d}(E, F)=\frac{\operatorname{dist}_{d}(E, F)}{\operatorname{dia}_{d}(E) \wedge \operatorname{dia}_{d}(F)} .
$$

Writing $P C(S)$ for the set of pairs of disjoint non-degenerate continua in $S \subset X$, a metric measure space $(X, d, \mu)$ is said to be $Q$-Loewner if for all $t>0$,

$$
\phi(t) \equiv \inf \left\{\bmod _{Q}(E, F):(E, F) \in P C(X), \Delta_{d}(E, F) \leq t\right\}>0 .
$$

We say that $(X, d, \mu)$ is locally $Q$-Loewner, or locally $\left(Q, \kappa, \delta_{0}, \psi\right)$ Loewner, if $\kappa \geq 1, \delta_{0} \in\left(0, \kappa^{-1}\right]$, and for all $t>0$,

$$
\begin{aligned}
& \psi(t) \equiv \inf \left\{\bmod _{Q}(E, F ; B(\kappa \delta d(x))):\right. \\
& \left.\quad(E, F) \in P C(B(x, \delta d(x))), \Delta_{d}(E, F) \leq t, x \in X, 0<\delta \leq \delta_{0}\right\}>0 .
\end{aligned}
$$

A typical example of a locally regular, locally Loewner space is an open set in a regular Loewner space; see [BoHeKo, Theorem 6.47].

The following lemma, in the case $r^{\prime}=r / 2$, is a restatement of Remark 3.19 in [HeKo]; our more general statement follows simply by chaining together paths generated by this special case.

Lemma 7.1. Suppose $(Y, d, \mu)$ is a bounded, $Q$-regular, $Q$-Loewner metric measure space. Then there exists a number $C_{0}>1$, dependent quantitatively on the hypotheses, such that if $x, y \in Y, x \neq y$, then $l(x, y)<C_{0} d(x, y)$. Moreover if $0<r^{\prime}<r, z \in Y$, and $x, y \in B_{d}(z, r) \backslash B_{d}\left(z, r^{\prime}\right)$, then there exists a path $\gamma \in \Gamma\left(x, y ; B_{d}\left(z, C_{0} r\right) \backslash B_{d}\left(z, r^{\prime} / C_{0}\right)\right)$ such that $\operatorname{len}_{d}(\gamma) \leq$ $C_{0} d(x, y)$.

Remark 7.2. The two concluded properties of $(Y, d)$ in Lemma 7.1 are inherited by $(\bar{Y}, \bar{d})$. This can be seen using a chaining argument in the case of both properties. Assume $x, y \in \bar{Y}$ are distinct. A path of length comparable to $\bar{d}(x, y)$ is constructed by chaining together paths between the points

$$
\ldots, x_{n+1}, x_{n}, \ldots, x_{1}, y_{1}, \ldots, y_{n}, y_{n+1}, \ldots
$$

where $\left(x_{n}\right),\left(y_{n}\right)$ are sequences of points in $Y$ that converge at a geometric rate to $x, y$, respectively. The second property is handled in a similar fashion, except that one should choose a point $z_{1} \in Y, \bar{d}\left(z_{1}, z\right)<r^{\prime} / 3 C_{0}$, and appropriate points $x_{n}, y_{n} \in B_{d}\left(z_{1}, 3 r / 2\right) \backslash B_{d}\left(z, 2 r^{\prime} / 3\right), n \in \mathbb{N}$, in order to construct a path in $\gamma \in \Gamma\left(x, y ; B_{d}\left(z, 2 C_{0} r\right) \backslash B_{d}\left(z, r^{\prime} / 3 C_{0}\right)\right)$.

The next lemma is of a well-known type, but for completeness we sketch the proof. 
Lemma 7.3. Suppose $(X, d, \mu)$ is a minimally nice, upper $Q$-regular, metric measure space, and let $C_{0}=2^{2 Q-1} C^{+}(\mu, Q)$. Then

$$
\bmod _{Q}(E, F) \leq C_{0}\left(\log _{2} \Delta_{d}(E, F)\right)^{-Q+1},
$$

whenever $E, F \subset X$ are disjoint non-degenerate compacta with $\Delta_{d}(E, F)$ $\geq 2$.

Proof. Without loss of generality, we assume that $r \equiv \operatorname{dia}_{d}(E) \leq \operatorname{dia}_{d}(F)$. Let us fix $x \in E$ and write $R=\operatorname{dist}_{d}(E, F)$, so that $\Delta_{d}(E, F)=R / r \geq 2$. Let $N=\left\lfloor\log _{2}(R / r)\right\rfloor$, and define a density

$$
\rho(z)= \begin{cases}1 /\left(2^{i-1} N r\right), & z \in \bar{B}_{d}\left(x, 2^{i} r\right) \backslash B_{d}\left(x, 2^{i-1} r\right), 1 \leq i \leq N, \\ 0, & \text { otherwise. }\end{cases}
$$

It is a rather routine task to verify that $\rho$ is an admissible weight and that for this choice of $\rho$ we have the inequality

$$
\int_{X} \rho^{Q} d \mu \leq C_{0}\left(\log _{2} \Delta_{d}(E, F)\right)^{-Q+1} .
$$

This estimate concludes the proof.

The next result is a rather technical modulus comparison lemma used only in the proof of Lemma 4.4, where $E$ is an annulus, $E^{\prime}$ is a ball concentric with $E$ that lies inside $E$, and $F$ is far from $E$.

Lemma 7.4. Let $(X, d, \mu)$ be a minimally nice, locally $\left(Q, \kappa, \delta_{0}, \psi\right)$ Loewner, metric measure space, let $E, E^{\prime}, F$ be three continua in $X$ with $0<\operatorname{dia}_{d}(E) \leq 3 \operatorname{dist}_{d}\left(E, E^{\prime}\right)$. Suppose further that $E^{\prime}$ lies in a component $G$ of $X \backslash E$, and that there exists some point $x \in X$, and a number $r \in\left(0, \delta_{0} d(x) / 4\right]$ such that $G \cup E \subset \overline{B_{d}}(x, r)$, and $F \subset X \backslash B_{d}(x, 3 r)$. Then

$$
\bmod _{Q}\left(E^{\prime}, F\right) \geq 3^{-Q}\left[\psi(3) \wedge \bmod _{Q}(E, F) \wedge \psi\left(\Delta_{d}\left(E, E^{\prime}\right)\right)\right] .
$$

Proof. Suppose $\int_{X} \rho^{Q}<3^{-Q}\left[\bmod _{Q}(E, F) \wedge \psi\left(\Delta_{d}\left(E, E^{\prime}\right)\right)\right]$ for some $\rho \in$ $A\left(E^{\prime}, F\right)$. Using the local Loewner condition, we have $\psi\left(\Delta_{d}\left(E, E^{\prime}\right)\right) \leq$ $\bmod _{Q}\left(E, E^{\prime}\right)$, and so $\int_{X}(3 \rho)^{Q}<\bmod _{Q}(E, F) \wedge \bmod _{Q}\left(E, E^{\prime}\right)$. Thus $3 \rho \notin$ $A(E, F) \cup A\left(E, E^{\prime}\right)$, and consequently there exist paths $\lambda_{1} \in \Gamma(F, E)$ and $\lambda_{2} \in \Gamma\left(E, E^{\prime}\right)$ such that $\int_{\lambda_{i}} \rho|d z|<1 / 3, i=1,2$.

We may assume that $\lambda_{2} \subset \bar{G}$, and that the domains of both $\lambda_{1}$ and $\lambda_{2}$ are $[0,1]$. Given a path $\eta$ from $\lambda_{1}\left(t_{1}\right)$ to $\lambda_{2}\left(t_{2}\right), t_{1}, t_{2} \in[0,1]$, we define a new path $\gamma=\left.\lambda_{1}\right|_{\left[0, t_{1}\right]}+\eta+\left.\lambda_{2}\right|_{\left[t_{2}, 1\right]}$. Since $\rho \in A\left(E^{\prime}, F\right)$ we have $\int_{\gamma} \rho \geq 1$ which implies that $\int_{\eta} \rho|d z| \geq 1-2 / 3=1 / 3$. Because $\eta \in \Gamma\left(\lambda_{1}, \lambda_{2}\right)$ was an arbitrary path we obtain that $3 \rho \in A\left(\lambda_{1}, \lambda_{2}\right)$. 
Let $\lambda_{3}$ be a segment of $\lambda_{1}$ which begins on $\partial B_{d}(x, 3 r)$ and ends in $E$. Then $\lambda_{2}$ and $\lambda_{3}$ are continua in $B_{d}\left(x, \delta_{0} d(x)\right)$, with $\operatorname{dia}_{d}\left(\lambda_{2}\right) \geq \operatorname{dist}_{d}\left(E, E^{\prime}\right) \geq$ $\operatorname{dia}_{d}(E) / 3, \operatorname{dia}_{d}\left(\lambda_{3}\right) \geq 2 r \geq \operatorname{dia}_{d} E$, and $\operatorname{dist}_{d}\left(\lambda_{2}, \lambda_{3}\right) \leq \operatorname{dia}_{d}(E)$. Thus

$$
\Delta_{d}\left(\lambda_{2}, \lambda_{3}\right) \leq \frac{\operatorname{dia}_{d}(E)}{\operatorname{dia}_{d}(E) / 3}=3,
$$

and so

$$
\bmod _{Q}\left(\lambda_{2}, \lambda_{3}\right) \geq \bmod _{Q}\left(\lambda_{2}, \lambda_{3} ; B\left(x, \delta_{0} \kappa d(x)\right)\right) \geq \psi(3) .
$$

But $3 \rho \in A\left(\lambda_{1}, \lambda_{2}\right) \subset A\left(\lambda_{3}, \lambda_{2}\right)$, and so $\int_{X} \rho^{Q} \geq 3^{-Q} \psi(3)$. The desired modulus estimate follows.

The following "metric quasiconformality implies geometric quasiconformality" type result of Tyson [Ty, Theorem 6.4] is needed in Sect. 4. It uses the notation

$$
\begin{aligned}
L_{f}(x, r) & =\sup \{d(f(x), f(y)): y \in \bar{B}(x, r)\}, \\
l_{f}(x, r) & =\inf \{d(f(x), f(z)): z \in X \backslash B(x, r)\} .
\end{aligned}
$$

Lemma 7.5. Let $X$ and $Y$ be locally compact metric measure spaces which are locally $Q$-regular for some $Q>1$. Let $f: X \rightarrow Y$ be a homeomorphism and let $H<\infty$ and $t>1$ be constants such that

$$
\forall x \in X: \quad \liminf _{r \rightarrow 0} \frac{L_{f}(x, t r)}{l_{f}(x, r)} \leq H .
$$

Then there exists $K$ dependent only in $t, H, Q$, and the $Q$-regularity constants of $X$ and $Y$ such that

$$
\bmod _{Q} \Gamma \leq K \bmod _{Q} f \Gamma,
$$

for every curve family $\Gamma$ in $X$.

\section{References}

[BaBo1] Balogh, Z.M., Bonk, M.: Pseudoconvexity and Gromov hyperbolicity. C.R. Acad. Sci., Paris, Sér. I, Math. 328, 597-602 (1999)

[BaBo2] Balogh, Z.M., Bonk, M.: Gromov hyperbolicity and the Kobayashi metric on strictly pseudoconvex domains. Comment. Math. Helv. 75, 504-533 (2000)

[BaBo3] Balogh, Z.M., Bonk, M.: Lengths of radii under conformal maps of the unit disc. Proc. Am. Math. Soc. 127, 801-804 (1999)

[Bo] Bonk, M.: Quasi-geodesic segments and Gromov hyperbolic spaces. Geom. Dedicata 62, 281-298 (1996)

[BoHeKo] Bonk, M., Heinonen, J., Koskela, P.: Uniformizing Gromov hyperbolic spaces. Astérisque 270

[BoKoRo] Bonk, M., Koskela, P., Rohde, S.: Conformal metrics in the unit ball in Euclidean space. Proc. Lond. Math. Soc., III. Ser. 77, 635-664 (1998) 
[BoSc] Bonk, M., Schramm, O.: Embeddings of Gromov hyperbolic spaces. Geom. Funct. Anal. 10, 266-306 (2000)

[BoPa] Bourdon, M., Pajot, H.: Poincaré inequalities and quasiconformal structure on the boundary of some hyperbolic buildings. Proc. Am. Math. Soc. 127, 2315-2324 (1999)

[BuKo1] Buckley, S.M., Koskela, P.: Sobolev-Poincaré implies John. Math. Res. Lett. 2, 577-593 (1995)

[BuKo2] Buckley, S.M., Koskela, P.: Criteria for Imbeddings of Sobolev-Poincaré type. Int. Math. Res. Not. no. 18, 881-901 (1996)

[BuOS] Buckley, S.M., O'Shea, J.: Weighted Trudinger-type inequalities. Indiana Univ. Math. J. 48, 85-114 (1999)

[BuSt1] Buckley, S.M., Stanoyevitch, A.: Weak slice conditions and Hölder imbeddings. J. Lond. Math. Soc., II. Ser. 66, 690-706 (2001)

[BuSt2] Buckley, S.M., Stanoyevitch, A.: Weak slice conditions, product domains, and quasiconformal mappings. Rev. Mat. Iberoam. 17, 1-37 (2001)

[CoDePa] Coornaert, M., Delzant, T., Papadopoulos, A.: Géometrie et théories des groupes. Lect. Notes Math. 1441. Berlin: Springer 1990

[Ge] Gehring, F.W.: Univalent functions and the Schwarzian derivative. Comment. Math. Helv. 52, 561-572 (1977)

[GeHa] Gehring, F.W., Hayman, W.K.: An inequality in the theory of conformal mapping. J. Math. Pures Appl., IX. Sér. 41, 353-361 (1962)

[GhHa] Ghys, E., de la Harpe, P. (eds.): Sur les groupes hyperboliques d'aprés Mikhael Gromov. Progress in Math. 38. Boston: Birkhäuser 1990

[Gr1] Gromov, M.: Hyperbolic Groups. Essays in Group Theory. S. Gersten (ed.), MSRI Publication, pp. 75-265. Springer 1987

[Gr2] Gromov, M.: Asymptotic invariants of infinite groups. Geometric Group Theory. Lond. Math. Soc. Lect. Not. Ser. 182 (1993)

[HeKo] Heinonen, J., Koskela, P.: Quasiconformal maps in metric spaces with controlled geometry. Acta Math. 181, 1-61 (1998)

[HeRo] Heinonen, J., Rohde, S.: The Gehring-Hayman theorem for quasihyperbolic geodesics. Math. Proc. Camb. Philos. Soc. 114, 393-405 (1993)

[Lk] Laakso, T.J.: Ahlfors $Q$-regular spaces with arbitrary $Q>1$ admitting weak Poincar inequality. Geom. Funct. Anal. 10, 111-123 (2000)

[Ln] Lang, U.: Extendability of large-scale Lipschitz maps. Trans. Am. Math. Soc. 351, 3975-3988 (1999)

[Ty] Tyson, J.T.: Metric and Geometric quasiconformality in Ahlfors regular Loewner soaces. Conform. Geom. Dyn. 5, 21-73 (2001)

[Va] Väisälä, J.: Relatively and inner uniform domains. Conform. Geom. Dyn. 2, 56-88 (1998) 